\title{
HOMOTOPY OF PRODUCT SYSTEMS AND $K$-THEORY OF CUNTZ-NICA-PIMSNER ALGEBRAS
}

\author{
JAMES FLETCHER, ELIZABETH GILLASPY, AND AIDAN SIMS
}

\begin{abstract}
We introduce the notion of a homotopy of product systems, and show that the Cuntz-Nica-Pimsner algebras of homotopic product systems over $\mathbb{N}^{k}$ have isomorphic $K$-theory. As an application, we give a new proof that the $K$-theory of a 2 -graph $C^{*}$ algebra is independent of the factorisation rules, and we further show that the $K$-theory of any twisted $k$-graph $C^{*}$-algebra is independent of the twisting 2-cocycle. We also explore applications to $K$-theory for the $C^{*}$-algebras of single-vertex $k$-graphs, reducing the question of whether the $K$-theory is independent of the factorisation rules to a question about path-connectedness of the space of solutions to an equation of YangBaxter type.
\end{abstract}

\section{INTRODUCTION}

The close link between the structure of a directed graph $E$ and that of its associated $C^{*}$-algebra $C^{*}(E)$, together with the structural restrictions on the $C^{*}$-algebras of directed graphs (for example [16], if $C^{*}(E)$ is simple, it must be either approximately finite-dimensional or purely infinite), spurred Kumjian and Pask to introduce higher-rank graphs ( $k$-graphs) in [15] as more general combinatorial models for $C^{*}$-algebras. Since then, substantial work has gone into the study of the structure of higher-rank graph $C^{*}$-algebras. Significant progress has been made on properties like simplicity [28], ideal structure [30, 31], pure infiniteness [31], stable finiteness and quasidiagonality [2], and topological dimension and real rank [23]. However, there has been little progress on calculating the $K$-theory of a $k$-graph $C^{*}$-algebra since the initial work of Robertson and Steger [29] on higher-rank Cuntz-Krieger algebras, and Evans' generalisation [4] of their work to the $C^{*}$-algebras of 2-graphs (higher-rank graphs of rank 2).

Higher-rank graphs, or $k$-graphs, are a $k$-dimensional generalization of directed graphs. One can view a $k$-graph as a directed graph with $k$ colours of edges, together with a factorisation rule which gives an equivalence relation on the set of paths in the graph: each path from a vertex $v$ to another vertex $w$ consisting of a red edge followed by a blue edge (a red-blue path) must be equivalent to precisely one blue-red path from $v$ to $w$. While the $C^{*}$-algebra of a $k$-graph depends on both the underlying edge-coloured directed graph (its skeleton) and on the factorisation rule, Evans' theorem shows that the $K$-theory of the $C^{*}$-algebra of a row-finite 2 -graph with no sources depends only on the skeleton. His techniques also suggest the more general possibility that the $K$-theory of any $k$-graph $C^{*}$-algebra is independent of the factorisation rules, and this was cast as a conjecture in [1]. Intriguingly, this question arose in [1] not through the study of $k$-graphs themselves, but because single-vertex $k$-graphs arise naturally as a framework to describe the "torsion

Date: November 5, 2019.

1991 Mathematics Subject Classification. 46L05.

Key words and phrases. Product system; Cuntz-Nica-Pimsner; higher-rank graph; K-theory. 
subalgebra" $\mathcal{A}_{\mathcal{S}}$ of the $C^{*}$-algebra $\mathcal{Q}_{S}$ associated to the multiplicative action on $\mathbb{Z}$ of the subsemigroup of $\mathbb{N}^{\times}$generated by a finite set $S$ of primes. In the single vertex case, every skeleton admits one set of factorisation rules under which the associated $C^{*}$-algebra is isomorphic to a tensor product $\bigotimes_{p \in S} \mathcal{O}_{p}$ of Cuntz algebras, but it also admits many other factorisation rules. Since the $C^{*}$-algebras $\mathcal{Q}_{S}$ and $\mathcal{A}_{S}$ are UCT Kirchberg algebras [1], the arguments of [1] reduce the question of understanding the $C^{*}$-algebras $\mathcal{Q}_{S}$ to the question of understanding $K_{*}\left(\mathcal{A}_{S}\right)$. It is therefore very interesting, even in the 1 -vertex case, to develop new techniques for investigating the conjecture that the $K$-theory of a $k$-graph $C^{*}$-algebra does not depend on its factorisation rules.

In this paper, we present an approach to this problem that reduces it to a question about path-connectedness of the space of unitary solutions to an equation of Yang-Baxter type (see, for example, [13]). Our idea is based on Elliott's technique [3] for computing the $K$-theory of a noncommutative torus. Elliott's technique exploits the fact that the noncommutative tori of a fixed rank assemble as the fibres of the $C^{*}$-algebra of a higherrank integer Heisenberg group. The base space for the fibration is a torus, hence path connected, so any two fibres can be connected by a path, putting them at either end of a $C([0,1])$-algebra. This $C([0,1])$-algebra can be viewed as a crossed product of $C([0,1])$ by $\mathbb{Z}^{k}$, and hence as an iterated crossed-product $C([0,1]) \rtimes \mathbb{Z} \rtimes \cdots \rtimes \mathbb{Z}$. Since evaluation at each point in $[0,1]$ induces an isomorphism $K_{*}(C([0,1])) \rightarrow K_{*}(\mathbb{C})$, it therefore suffices, by induction, to prove that if $A$ is a $C([0,1])$-algebra such that the quotient maps $A \mapsto A_{t}$ induce isomorphisms in $K$-theory, and if $\alpha$ is an automorphism of $A$ that respects the $C([0,1])$-algebra structure, then $A \rtimes \mathbb{Z}$ is again a $C([0,1])$-algebra in which the evaluation maps induce isomorphisms in $K$-theory. Elliott proved this by applying the Five Lemma to the Pimsner-Voiculescu exact sequence for the crossed product. A similar technique was used in [20, 10] to examine the $K$-theory of twisted $k$-graph $C^{*}$-algebras, and show that homotopic cocycles yield twisted $k$-graph $C^{*}$-algebras with isomorphic $K$-theory.

Here, we employ a similar technique, but expand the notion of homotopy to which our results apply by regarding product systems of Hilbert modules as generalised cocycles. Product systems (see Section 2.1 below) and their $C^{*}$-algebras were introduced by Fowler in [7]. These families $\left\{X_{p}\right\}_{p \in P}$ of Hilbert $A-A$-bimodules, indexed by a semigroup $P$ and carrying a multiplication compatible with that of $P$, give rise to a class of $C^{*}$-algebras including $k$-graph $C^{*}$-algebras and crossed products by endomorphisms.

We introduce homotopies of product systems with coefficient algebra $A$, defined as product systems $X$ with coefficient algebra $C([0,1], A)$ in which the canonical left and right actions of $C([0,1])$ on each fibre coincide. Proposition 3.4 verifies that this notion of homotopy defines an equivalence relation on (isomorphism classes of) product systems, while Lemma 3.1 establishes that a homotopy $X$ of product systems decomposes into fibres $X^{t}$ indexed by $t \in[0,1]$ such that each $X^{t}$ is itself a product system over $A$. Lemma 3.1 also establishes that if the left action on a homotopy of product systems is injective and/or by compacts then the same is true in each fibre. Using this, we verify in Proposition 3.5 that for product systems over a quasi-lattice ordered semigroup in which each pair of elements has an upper bound, the Cuntz-Nica-Pimsner algebra of a homotopy of product systems in which the left action is injective and by compacts itself decomposes as a $C([0,1])$-algebra whose fibres are the Cuntz-Nica-Pimsner algebras of the fibres of the homotopy. We then focus on product systems over $\mathbb{N}^{k}$, and use Elliott's strategy 
described above, replacing the Pimsner-Voiculescu exact sequence with Pimsner's sixterm exact sequence for Cuntz-Pimsner algebras [24], to show in Theorem 3.7 that if $X$ is a homotopy of product systems over $\mathbb{N}^{k}$, then the quotient maps $\mathcal{N} \mathcal{O}_{X} \rightarrow \mathcal{N} \mathcal{O}_{X^{t}}$ all induce isomorphisms in $K$-theory.

We conclude the paper by applying this machinery to the setting of $k$-graphs. We use results of [9] to show that given a system $E_{1}, \ldots, E_{k}$ of row-finite directed graphs that could arise as the skeleton of a $k$-graph (where $E_{i}$ consists of the edges of colour $i$ ), product systems over $\mathbb{N}^{k}$ with coordinate fibres isomorphic to the graph modules $X\left(E_{i}\right)$ are determined by systems of unitary matrices $U_{i, j}(v, w): \mathbb{C}^{v E_{j}^{1} E_{i}^{1} w} \rightarrow \mathbb{C}^{v E_{i}^{1} E_{j}^{1} w}$, indexed by pairs $v, w$ of vertices and pairs $1 \leq i<j \leq k$ of indices, that collectively satisfy a cocycle condition reminiscent of the Yang-Baxter equation. We call such a system a unitary cocycle for $E$. For example, Proposition 4.7 establishes that for every row-finite $k$-graph $\Lambda$ with no sources, and every $\mathbb{T}$-valued 2-cocycle $c$ on $\Lambda$, there is a unitary cocycle so that the Cuntz-Nica-Pimsner algebra of the associated product system coincides with the twisted $k$-graph $C^{*}$-algebra $C^{*}(\Lambda, c)$. We then show, in Proposition 4.9, that a family $\left\{U^{t}: t \in[0,1]\right\}$ of unitary cocycles for $E$ in which, for each fixed $u, v, i, j$, the map $t \mapsto U_{i, j}^{t}(u, v)$ is continuous, determines a homotopy of product systems. We call such a family a continuous path of unitary cocycles for $E$. Using our main result, we then deduce (Corollary 4.10) that if $\Lambda, \Gamma$ are $k$-graphs with the same skeleton $E$, and $c, c^{\prime}$ are $\mathbb{T}$-valued cocycles on $\Lambda$ and $\Gamma$, and if the unitary cocycles for $E$ determined by $(\Lambda, c)$ and $\left(\Gamma, c^{\prime}\right)$ are connected by a continuous path of unitary cocycles for $E$, then $C^{*}(\Lambda, c)$ and $C^{*}\left(\Gamma, c^{\prime}\right)$ have isomorphic $K$-theory.

We deduce two main consequences. First, if $E=\left(E_{1}, E_{2}\right)$ is a skeleton of a 2-graph, then the cocycle condition for a unitary cocycle for $E$ is vacuous, and so a unitary cocycle is simply a system of unitary isomorphisms $U_{1,2}(v, w)$, indexed by $v, w \in E^{0}$, of finitedimensional vector spaces. Since the space of $n \times n$ complex unitary matrices is pathconnected, we deduce that the space of unitary cocycles for $E$ is path connected, and therefore that any two product systems over $\mathbb{N}^{2}$ with coordinate fibres $X\left(E_{1}\right)$ and $X\left(E_{2}\right)$ are connected by a homotopy of product systems. Hence the $K$-theory of the CuntzNica-Pimsner algebra of any such product system is determined by Evans' calculation of $K$-theory for 2-graph $C^{*}$-algebras. In particular, if $\Lambda$ and $\Gamma$ are row-finite 2-graphs with no sources and the same skeleton, then $K_{*}\left(C^{*}(\Lambda, c)\right) \cong K_{*}\left(C^{*}\left(\Gamma, c^{\prime}\right)\right)$ for any $\mathbb{T}$ valued cocycles $c$ on $\Lambda$ and $c^{\prime}$ on $\Lambda^{\prime}$. Second, given positive integers $n_{1}, \ldots, n_{k}$, a unitary cocycle for the $k$-skeleton $\left(E_{1}, \ldots E_{k}\right)$ in which each graph $E_{i}$ has one vertex and $n_{i}$ edges reduces to a family of unitary matrices $U_{i, j}: \mathbb{C}^{n_{j}} \otimes \mathbb{C}^{n_{i}} \rightarrow \mathbb{C}^{n_{i}} \otimes \mathbb{C}^{n_{j}}, i<j$, satisfying the Yang-Baxter type equations

$$
\left(U_{i, j} \otimes 1_{l}\right)\left(1_{j} \otimes U_{i, l}\right)\left(U_{j, l} \otimes 1\right)=\left(1_{i} \otimes U_{j, l}\right)\left(U_{i, l} \otimes 1_{j}\right)\left(1_{l} \otimes U_{i, j}\right)
$$

for $1 \leq i<j<l \leq k$. In particular, if the collection of all such families of unitary matrices is connected, then any twisted $C^{*}$-algebra of any 1 -vertex $k$-graph $\Lambda$ in which each $\left|\Lambda^{e_{i}}\right|=n_{i}$ has $K$-theory isomorphic to $K_{*}\left(\bigotimes_{i=1}^{k} \mathcal{O}_{n_{i}}\right)$.

The structure of the paper is as follows. We introduce background on Hilbert bimodules and on product systems in Section 2, In Section 3 we introduce the notion of homotopy of product systems, and prove our main result, Theorem 3.7, which states that the Cuntz-Nica-Pimsner algebras of homotopic product systems have isomorphic $K$-theory. In Section 4 we present background on $k$-graphs and their twisted $C^{*}$-algebras, 
introduce the notion of a unitary cocycle for a $k$-skeleton $E$, and use this to apply our earlier results to twisted $k$-graph $C^{*}$-algebras.

Throughout the paper, we use the word "homomorphism" to denote a *-preserving, multiplicative, norm-decreasing linear map between $C^{*}$-algebras.

\section{Background on Hilbert Bimodules and associated $C^{*}$-algebras}

We give a quick summary of the structure of Hilbert modules and their $C^{*}$-algebras. For details on Hilbert modules, see [22, 27] and for details on the associated $C^{*}$-algebras, see [24, 8, 14].

Let $A$ be a $C^{*}$-algebra. An inner product $A$-module is a complex vector space $X$ equipped with a map $\langle\cdot, \cdot\rangle_{A}: X \times X \rightarrow A$, linear in its second argument, and a right action of $A$, such that for any $x, y \in X$ and $a \in A$, we have

(i) $\langle x, y\rangle_{A}=\langle y, x\rangle_{A}^{*}$;

(ii) $\langle x, y \cdot a\rangle_{A}=\langle x, y\rangle_{A} a$;

(iii) $\langle x, x\rangle_{A} \geq 0$ in $A$; and

(iv) $\langle x, x\rangle_{A}=0$ if and only if $x=0$.

By [22, Proposition 1.1], the formula $\|x\|_{X}:=\left\|\langle x, x\rangle_{A}\right\|_{A}^{1 / 2}$ defines a norm on $X$, and we say that $X$ is a Hilbert $A$-module if $X$ is complete with respect to this norm.

We say that a map $T: X \rightarrow X$ is adjointable if there exists a map $T^{*}: X \rightarrow X$ such that $\langle T x, y\rangle_{A}=\left\langle x, T^{*} y\right\rangle_{A}$ for all $x, y \in X$. Every adjointable operator $T$ is automatically linear and continuous, and the adjoint $T^{*}$ is unique. Equipping the collection of adjointable operators on $X$, denoted by $\mathcal{L}(X)$, with the operator norm gives a $C^{*}$-algebra. For each $x, y \in X$, the formula $\Theta_{x, y}(z):=x \cdot\langle y, z\rangle_{A}$ defines an adjointable operator with adjoint $\Theta_{x, y}^{*}=\Theta_{y, x}$. The closed subspace $\mathcal{K}(X):=\overline{\operatorname{span}}\left\{\Theta_{x, y}: x, y \in X\right\}$ is an essential ideal of $\mathcal{L}(X)$, whose elements we refer to as compact operators.

A Hilbert $A$-bimodule is a Hilbert $A$-module $X$ equipped with a left action of $A$ by adjointable operators (i.e. a homomorphism $\phi: A \rightarrow \mathcal{L}(X)$ ). We frequently write $a \cdot x$ for $\phi(a)(x)$. Letting $A$ act on itself by left and right multiplication, and defining an $A$-valued inner product on $A$ by $\langle a, b\rangle_{A}:=a^{*} b$, gives a Hilbert $A$-bimodule that we denote by ${ }_{A} A_{A}$.

By the Hewitt-Cohen factorisation theorem, every Hilbert $A$-bimodule $X$ is automatically right-nondegenerate in the strong sense that $X=X \cdot A$. So we will say that the Hilbert $A$-bimodule is nondegenerate if the homomorphism $\phi: A \rightarrow \mathcal{L}(X)$ that implements the left action is nondegenerate; that is if $X=\overline{\phi(A) X}$.

Example 2.1. Let $E=\left(E^{0}, E^{1}, r, s\right)$ be a row-finite graph with no sources. Let $A:=$ $C_{0}\left(E^{0}\right)$. Define $\langle\cdot, \cdot\rangle_{A}$ on $C_{c}\left(E^{1}\right)$ by $\langle\xi, \eta\rangle_{A}(v)=\sum_{e \in E^{1} v} \overline{\xi(e)} \eta(e)$, and define left and right actions of $A$ on $C_{c}\left(E^{1}\right)$ by $(a \cdot \xi \cdot b)(e)=a(r(e)) \xi(e) b(s(e))$. Then $\|\xi\|:=\left\|\langle\xi, \xi\rangle_{A}\right\|^{1 / 2}$ defines a norm on $C_{c}\left(E^{1}\right)$. The completion $X=X(E)$ of $C_{c}(E)$ in this norm is a Hilbert $A$-bimodule with $A$-actions extending those on $C_{c}\left(E^{1}\right)$. This is called the graph bimodule of $E$.

A Toeplitz representation $(\psi, \pi)$ of a Hilbert $A$-bimodule $X$ in a $C^{*}$-algebra $B$ consists of a linear map $\psi: X \rightarrow B$ and a homomorphism $\pi: A \rightarrow B$ such that

(i) $\psi(a \cdot x)=\pi(a) \psi(x)$ for each $a \in A, x \in X$;

(ii) $\psi(x)^{*} \psi(y)=\pi\left(\langle x, y\rangle_{A}\right)$ for each $x, y \in X$. 
These relations imply that $\psi(x \cdot a)=\psi(x) \pi(a)$ for all $a \in A$ and $x \in X$, and also that $\psi$ is norm-decreasing, and is isometric if and only $\pi$ is injective on $\langle X, X\rangle_{A}$. The universal $C^{*}$ algebra for Toeplitz representations of $X$ is called the Toeplitz algebra of $X$. We write $\mathcal{T}_{X}$ for this $C^{*}$-algebra and denote the universal Toeplitz representation of $X$ that generates it by $\left(i_{X}, i_{A}\right)$. By [25, Proposition 8.11], if $(\psi, \pi)$ is a Toeplitz representation of $X$ in $B$, then there is a homomorphism $(\psi, \pi)^{(1)}: \mathcal{K}(X) \rightarrow B$ such that $(\psi, \pi)^{(1)}\left(\Theta_{x, y}\right)=\psi(x) \psi(y)^{*}$ for all $x, y \in X$. We say that a Toeplitz representation $(\psi, \pi)$ is Cuntz-Pimsner covariant if $(\psi, \pi)^{(1)}(\phi(a))=\pi(a)$ for all $\left.a \in J_{X}:=\phi^{-1}(\mathcal{K}(X)) \cap \operatorname{ker}(\phi)^{\perp}\right]$ We call the universal $C^{*}$ algebra for Cuntz-Pimsner covariant Toeplitz representations of $X$ the Cuntz-Pimsner algebra of $X$. We denote this $C^{*}$-algebra by $\mathcal{O}_{X}$ and write $\left(j_{X}, j_{A}\right)$ for the universal Cuntz-Pimsner covariant Toeplitz representation of $X$.

The $K$-theory of the Toeplitz algebra of a Hilbert $A$-bimodule $X$ is easy to compute: by [14, Proposition 8.2] the homomorphism $i_{A}: A \rightarrow \mathcal{T}_{X}$ induces an isomorphism at the level of $K$-theory (in fact if $A$ is separable and $X$ is countably generated, then this homomorphism induces a $K K$-equivalence [24, Theorem 4.4]). In general, the $K$-theory of the Cuntz-Pimsner algebra $\mathcal{O}_{X}$ is much more complicated; the primary tool for computing it is the following 6-term exact sequence [14, Theorem 8.6]

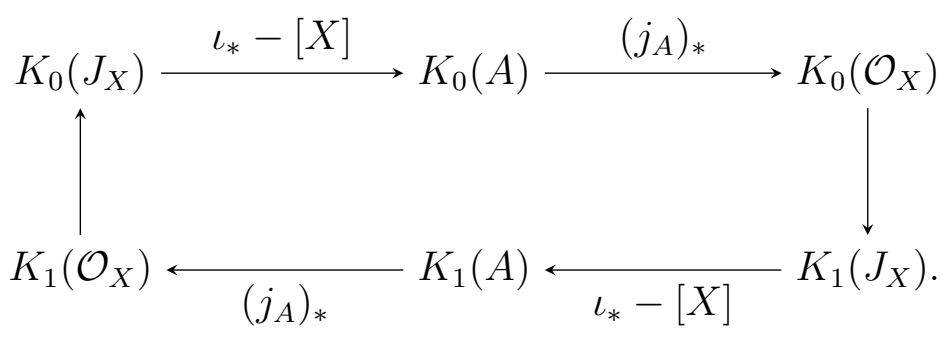

Given Hilbert $A$-bimodules $X$ and $Y$, we can form the balanced tensor product $X \otimes_{A} Y$ as follows (see [22] or [27]). We endow the algebraic tensor product $X \odot Y$ with the canonical actions of $A$ given by $a \cdot(x \odot y) \cdot b=(a \cdot x) \odot(y \cdot b)$, and with the sesquilinear form $\left[x \odot y, x^{\prime} \odot y^{\prime}\right]_{A}:=\left\langle y,\left\langle x, x^{\prime}\right\rangle_{A} \cdot y^{\prime}\right\rangle_{A}$. The space $N=\left\{\zeta \in X \odot Y:[\zeta, \zeta]_{A}=0\right\}$ is a closed submodule, and $[\cdot, \cdot]_{A}$ descends to an inner-product $\langle\cdot, \cdot\rangle_{A}$ on $(X \odot Y) / N$. The balanced tensor product $X \otimes_{A} Y$ is the completion of $(X \odot Y) / N$ in the inner-product norm, which is itself a Hilbert $A$-bimodule. We write $x \otimes y$ for the image $x \odot y+N$ of $x \odot y$ in $X \otimes_{A} Y$. A simple calculation shows that $[x \cdot a \odot y, \zeta]_{A}=[x \odot a \cdot y, \zeta]$ for all $x \in X, y \in Y, a \in A$ and $\zeta \in X \odot Y$, and it follows that $x \cdot a \otimes y=x \otimes a \cdot y$ for all $x, y, a$. If $\phi: A \rightarrow \mathcal{L}(Y)$ is the homomorphism implementing the left action, we sometimes write $X \otimes_{\phi} Y$ rather than $X \otimes_{A} Y$.

We define the balanced tensor powers of $X$ as follows: $X^{\otimes 0}:={ }_{A} A_{A}, X^{\otimes 1}:=X$, and $X^{\otimes n}:=X \otimes_{A} X^{\otimes n-1}$ for $n \geq 2$. Given Hilbert $A$-bimodules $X$ and $Y$ and an adjointable operator $S \in \mathcal{L}(X)$, the formula $x \otimes y \mapsto(S x) \otimes y$ extends to an adjointable operator on all of $X \otimes_{A} Y$, which we denote by $S \otimes_{A} 1_{Y}$, or by $S \otimes_{\phi} 1_{Y}$ if $\phi: A \rightarrow \mathcal{L}(X)$ is the homomorphism implementing the left action.

We can also combine a collection $\left\{X_{j}: j \in J\right\}$ of Hilbert $A$-bimodules by forming their direct sum. We define $\bigoplus_{j \in J} X_{j}$ to be the set of sequences $\left(x_{j}\right)_{j \in J}$ with $x_{j} \in X_{j}$, such that $\sum_{j \in J}\left\langle x_{j}, x_{j}\right\rangle_{A}$ converges in $A$. We define an $A$-valued inner product on $\bigoplus_{j \in J} X_{j}$ by $\left\langle\left(x_{j}\right)_{j \in J},\left(y_{j}\right)_{j \in J}\right\rangle_{A}:=\sum_{j \in J}\left\langle x_{j}, y_{j}\right\rangle_{A}$, which converges by [22, Proposition 1.1]. It follows

\footnotetext{
${ }^{1}$ Given an ideal $I$ of a $C^{*}$-algebra $A$, we write $I^{\perp}$ for the annihilator $\{a \in A: a I=0\}$.
} 
that $\bigoplus_{j \in J} X_{j}$ is complete with respect to the norm induced by this inner product. Letting $A$ act componentwise on the left and right then gives $\bigoplus_{j \in J} X_{j}$ the structure of a Hilbert A-bimodule.

2.1. Product systems of Hilbert bimodules and their associated $C^{*}$-algebras. A quasi-lattice ordered group $(G, P)$ consists of a group $G$ and a subsemigroup $P$ of $G$ such that $P \cap P^{-1}=\{e\}$, and such that, with respect to the partial order on $G$ given by $p \leq q \Leftrightarrow p^{-1} q \in P$, any two elements $p, q \in G$ which have a common upper bound in $P$ have a least common upper bound $p \vee q$ in $P$. We write $p \vee q=\infty$ if $p$ and $q$ have no common upper bound in $P$, and $p \vee q<\infty$ otherwise. We say that $P$ is directed if $p \vee q<\infty$ for every $p, q \in P$.

Let $(G, P)$ be a quasi-lattice ordered group and $A$ a $C^{*}$-algebra. A product system over $P$ with coefficient algebra $A$ is a semigroup $X=\bigsqcup_{p \in P} X_{p}$ such that:

(i) $X_{e}={ }_{A} A_{A}$, and $X_{p} \subseteq X$ is a Hilbert $A$-bimodule for each $p \in P$;

(ii) for each $p, q \in P$ with $p \neq e$, there exists a Hilbert $A$-bimodule isomorphism $M_{p, q}: X_{p} \otimes_{A} X_{q} \rightarrow X_{p q}$ which is associative in the sense that

$$
M_{r, p q} \circ 1_{X_{r}} \otimes M_{p, q}=M_{r p, q} \circ M_{r, p} \otimes 1_{X_{q}}
$$

for each $p, q, r \in P$; and

(iii) multiplication in $X$ by elements of $X_{e}=A$ implements the left and right actions of $A$ on each $X_{p}$; that is $x a=x \cdot a$ and $a x=a \cdot x$ for each $p \in P, a \in A$, and $x \in X_{p}$.

We will often write $M_{p, q}(x \otimes y)=: x y$.

We will say that the product system $X$ is nondegenerate if each fibre $X_{p}$ is nondegenerate as a Hilbert $A$-bimodule; that is, if $X_{p}=\overline{\phi_{p}(A) X_{p}}$ for all $p$. Note that since the multiplication maps $X_{e} \times X_{p} \rightarrow X_{p}$ are given by the left action, the product system $X$ is nondegenerate if and only if condition (ii) above also holds when $p=e$.

We write $\phi_{p}: A \rightarrow \mathcal{L}\left(X_{p}\right)$ for the homomorphism that implements the left action of $A$ on $X_{p}$. Since multiplication in $X$ is associative, $\phi_{p q}(a)(x y)=\left(\phi_{p}(a) x\right) y$ for all $p, q \in P$, $a \in A, x \in X_{p}$, and $y \in X_{q}$. We write $\langle\cdot, \cdot\rangle_{A}^{p}$ for the $A$-valued inner-product on $X_{p}$.

For each $p, q \in P$, we define a homomorphism $\iota_{p}^{p q}: \mathcal{L}\left(X_{p}\right) \rightarrow \mathcal{L}\left(X_{p q}\right)$ by

$$
\iota_{p}^{p q}(S):=M_{p, q} \circ\left(S \otimes_{A} 1_{X_{q}}\right) \circ M_{p, q}^{-1}
$$

for each $S \in \mathcal{L}\left(X_{p}\right)$. Equivalently, $\iota_{p}^{p q}$ is characterised by the formula $\iota_{p}^{p q}(S)(x y)=(S x) y$ for each $S \in \mathcal{L}\left(X_{p}\right), x \in X_{p}, y \in X_{q}$. We define $\iota_{p}^{r}: \mathcal{L}\left(X_{p}\right) \rightarrow \mathcal{L}\left(X_{r}\right)$ to be the zero map whenever $p \not \leq r$. We say that $X$ is compactly aligned if $\iota_{p}^{p \vee q}(S) \iota_{q}^{p \vee q}(T) \in \mathcal{K}\left(X_{p \vee q}\right)$ whenever $S \in \mathcal{K}\left(X_{p}\right)$ and $T \in \mathcal{K}\left(X_{q}\right)$ for some $p, q \in P$ with $p \vee q<\infty$. By [7, Proposition 5.8], if $A$ acts compactly on each fibre of $X$ (i.e. $\phi_{p}(A) \subseteq \mathcal{K}\left(X_{p}\right)$ for each $p \in P$ ) or $(G, P)$ is totally ordered by $\leq$, then $X$ is automatically compactly aligned.

A representation of a compactly aligned product system $X$ over $P$ in a $C^{*}$-algebra $B$ is a map $\psi: X \rightarrow B$ such that

(i) each $\psi_{p}:=\left.\psi\right|_{X_{p}}$ is a linear map, and $\psi_{e}$ is a homomorphism;

(ii) $\psi_{p}(x) \psi_{q}(y)=\psi_{p q}(x y)$ for all $p, q \in P$ and $x \in X_{p}, y \in X_{q}$; and

(iii) $\psi_{p}(x)^{*} \psi_{p}(y)=\psi_{e}\left(\langle x, y\rangle_{A}^{p}\right)$ for all $p \in P$ and $x, y \in X_{p}$.

For each $p \in P$, it follows that $\left(\psi_{p}, \psi_{e}\right)$ is a Toeplitz representation of the Hilbert $A$ bimodule $X_{p}$. We write $\psi^{(p)}$ for the resulting homomorphism $\left(\psi_{p}, \psi_{e}\right)^{(1)}: \mathcal{K}\left(X_{p}\right) \rightarrow B$. 
If $\rho: B \rightarrow C$ is a homomorphism of $C^{*}$-algebras and $\psi: X \rightarrow B$ is a representation, then $\rho \circ \psi: X \rightarrow C$ is a representation. For $x, y \in X_{p}$, we have $(\rho \circ \psi)^{(p)}\left(\theta_{x, y}\right)=$ $\rho(\psi(x)) \rho(\psi(y))^{*}=\rho\left(\psi(x) \psi(y)^{*}\right)=\rho \circ \psi^{(p)}\left(\theta_{x, y}\right)$. So linearity and continuity give

$$
(\rho \circ \psi)^{(p)}=\rho \circ \psi^{(p)} \text {. }
$$

We say that $\psi$ is Nica covariant if, for any $p, q \in P$ and $S \in \mathcal{K}\left(X_{p}\right), T \in \mathcal{K}\left(X_{q}\right)$,

$$
\psi^{(p)}(S) \psi^{(q)}(T)= \begin{cases}\psi^{(p \vee q)}\left(\iota_{p}^{p \vee q}(S) \iota_{q}^{p \vee q}(T)\right) & \text { if } p \vee q<\infty \\ 0 & \text { otherwise. }\end{cases}
$$

We denote the universal $C^{*}$-algebra for Nica covariant representations by $\mathcal{N} \mathcal{T}_{X}$ (the $N i c a^{-}$ Toeplitz algebra of $X$ ) and write $\left\{i_{p}: X_{p} \rightarrow \mathcal{N} \mathcal{T}_{X}\right\}_{p \in P}$ for its generating representation. It then follows from relations (i)-(iii) that $\mathcal{N} \mathcal{T}_{X}=\overline{\operatorname{span}}\left\{i_{X}(x) i_{X}(y)^{*}: x, y \in X\right\}$.

For representations of product systems we also have a notion of Cuntz-Pimsner covariance (first introduced by Sims and Yeend in [32]). To formulate this covariance relation we first require some additional definitions. We start by defining a collection of ideals of $A$ by setting $I_{e}:=A$ and $I_{p}:=\bigcap_{e<q \leq p} \operatorname{ker}\left(\phi_{q}\right)$ for each $p \in P \backslash\{e\}$. For each $p \in P$, we then define a Hilbert $A$-bimodule

$$
\widetilde{X}_{p}:=\bigoplus_{q \leq p} X_{q} \cdot I_{q^{-1} p}
$$

and write $\widetilde{\phi}_{p}: A \rightarrow \mathcal{L}\left(\widetilde{X}_{p}\right)$ for the homomorphism defined by

$$
\left(\widetilde{\phi}_{p}(a)(x)\right)_{q}:=\phi_{q}(a)\left(x_{q}\right) \text { for } a \in A, x \in \widetilde{X}_{p}, q \leq p .
$$

We say that $X$ is $\widetilde{\phi}$-injective if each homomorphism $\widetilde{\phi}_{p}$ is injective. For various examples (in particular, for product systems over $\mathbb{N}^{k}$, or where each $\phi_{p}$ is injective) $\widetilde{\phi}$-injectivity is automatic by [32, Lemma 3.15]. For each $p, q \in P$, we have a homomorphism $\widetilde{\iota}_{p}^{q}$ : $\mathcal{L}\left(X_{p}\right) \rightarrow \mathcal{L}\left(\widetilde{X}_{q}\right)$ characterised by the formula

$$
\left(\widetilde{\iota}_{p}^{q}(S)(x)\right)_{r}=\iota_{p}^{r}(S)\left(x_{r}\right) \quad \text { for } S \in \mathcal{L}\left(X_{p}\right), x \in \widetilde{X}_{p}, r \leq q .
$$

Given a predicate statement $\mathcal{P}(s)$ (where $s \in P$ ), we say that $\mathcal{P}(s)$ is true for large $s$ if, given any $p \in P$, there exists $q \geq p$, such that $\mathcal{P}(s)$ is true whenever $s \geq q$. We then say that a Nica covariant representation $\psi$ of a compactly aligned $\widetilde{\phi}$-injective product system $X$ is Cuntz-Pimsner covariant if, for any finite set $F \subseteq P$ and any choice of compact operators $\left\{T_{p} \in \mathcal{K}\left(X_{p}\right): p \in F\right\}$, we have that

$$
\sum_{p \in F} \widetilde{\iota}_{p}^{s}\left(T_{p}\right)=0 \in \mathcal{L}\left(\widetilde{X}_{s}\right) \quad \text { for large } s \Rightarrow \sum_{p \in F} \psi^{(p)}\left(T_{p}\right)=0 .
$$

We say that $\psi$ is Cuntz-Nica-Pimsner covariant if it is both Nica covariant and CuntzPimsner covariant.

If $P$ is directed and each $\phi_{p}$ is injective and takes values in $\mathcal{K}\left(X_{p}\right)$, then [32, Corollary 5.2] shows that a representation $\psi$ of $X$ is Cuntz-Nica-Pimsner covariant if and only if $\psi^{(p)} \circ \phi_{p}=\psi_{e}$ for each $p \in P$.

We denote the universal $C^{*}$-algebra for Cuntz-Nica-Pimsner covariant covariant representations by $\mathcal{N O}_{X}$ (the Cuntz-Nica-Pimsner algebra of $X$ ) and write $\left\{j_{p}: X_{p} \rightarrow\right.$ $\left.\mathcal{N} \mathcal{O}_{X}\right\}_{p \in P}$ for its generating representation. It follows that $\mathcal{N} \mathcal{O}_{X}$ is a quotient of $\mathcal{N} \mathcal{T}_{X}$, 
and we write $q: \mathcal{N} \mathcal{T}_{X} \rightarrow \mathcal{N O}_{X}$ for the quotient homomorphism (characterised by $\left.q \circ i_{X}=j_{X}\right)$. We have $\mathcal{N} \mathcal{O}_{X}=\overline{\operatorname{span}}\left\{j_{X}(x) j_{X}(y)^{*}: x, y \in X\right\}$.

\section{Homotopies of PRODUCT Systems}

To define our notion of a homotopy of product systems, we begin with a little background. Suppose that $A$ is a $C^{*}$-algebra, and that $X$ is a right-Hilbert $(A \otimes C([0,1]))$ module. Identifying $A \otimes C([0,1])$ with $C([0,1], A)$ as usual, Cohen factorisation shows that each $x \in X$ can be written as $x=y \cdot f$ for some $y \in X$ and $f \in C([0,1], A)$. It follows that there is an action of $C([0,1])$ on the right of $X$ such that $(x \cdot f) \cdot g=x \cdot(f g)$ for all $x \in X, f \in C([0,1], A)$ and $g \in C([0,1])$.

With $X$ and $A$ as above, for each $t \in[0,1]$, we write $I_{t}$ for the ideal $A \otimes C_{0}([0,1] \backslash\{t\}) \subseteq$ $A \otimes C([0,1])$, and we write $X^{t}$ for the quotient right-Hilbert $A$-module $X /\left(X \cdot I_{t}\right)$. It is standard that there is a unique topology on $\mathcal{X}:=\bigsqcup_{t} X^{t}$ under which $\mathcal{X}$ is a continuous Banach bundle in which, for each $x \in X$, the map

$$
\gamma_{x}: t \mapsto x+X \cdot I_{t}
$$

is continuous. With respect to this topology, the map $X \ni x \mapsto \gamma_{x} \in \Gamma([0,1], \mathcal{X})$ is an isomorphism of $X$ onto the module of continuous sections of $\mathcal{X} 2$

Now suppose that $X$ is a nondegenerate Hilbert bimodule over $A \otimes C([0,1])$. Then $\phi$ extends to a homomorphism from $\mathcal{M}(A \otimes C([0,1]))=\mathcal{M}(A) \otimes C([0,1])$ to $\mathcal{L}(X)$, and in particular determines a left action of $C([0,1])$ on $X$. We say that $X$ is fibred over $[0,1]$ if $f \cdot x=x \cdot f$ for all $x \in X$ and $f \in C([0,1])$. If $X$ is fibred over $[0,1]$, then for each $t \in[0,1]$, the right-Hilbert module $X^{t}$ becomes a Hilbert $A$-bimodule with left action satisfying $a \cdot\left(x+I_{t}\right)=(f \cdot x)+I_{t}$ for any $f \in C([0,1], A)$ satisfying $f(t)=a$.

If $P$ is a semigroup, and $X$ is a product system over $P$ with coefficient algebra $A \otimes$ $C([0,1])$, we will say that $X$ is fibred over $[0,1]$ if each $X_{p}$ is fibred over $[0,1]$.

Lemma 3.1. Let $P$ be a semigroup, and let $X$ be a nondegenerate product system over $P$ with coefficient algebra $C([0,1], A)$ that is fibred over $[0,1]$. For each $t \in[0,1]$ the system $X^{t}:=\left\{X_{p}^{t}: p \in P\right\}$ is a product system over $P$ with coefficient algebra $A$, with multiplication given by $\left(x+X_{p} \cdot I_{t}\right)\left(y+X_{q} \cdot I_{t}\right)=x y+X_{p q} \cdot I_{t}$ for all $p, q \in P, x \in X_{p}$ and $y \in X_{q}$. For any $p \in P$, if the left action of $C([0,1], A)$ on $X_{p}$ is by compacts, then the left action of $A$ on each $X_{p}^{t}$ is by compacts. For any $p \in P$, if the action of $A$ on each $X_{p}^{t}$ is faithful then the action of $C([0,1], A)$ on $X_{p}$ is faithful; and if the left action of $C([0,1], A)$ on $X_{p}$ is by compacts, then the converse holds.

Proof. Fix $t \in[0,1]$. Each $X_{t}$ is a Hilbert $A$-bimodule as discussed above, so we just have to show that the formula $\left(x+X_{p} \cdot I_{t}\right)\left(y+X_{q} \cdot I_{t}\right)=x y+X_{p q} \cdot I_{t}$ determines a well-defined multiplication that induces isomorphisms $X_{p}^{t} \otimes_{A} X_{q}^{t} \cong X_{p q}^{t}$. For $p, q \in P$ let $M_{p, q}: X_{p} \otimes_{C([0,1], A)} X_{q} \rightarrow X_{p q}$ be the multiplication map. Fix $x \in X_{p} \cdot I_{t}$, and write $x=x^{\prime} \cdot f$ where $f(t)=0$. Since $M_{p, q}$ is an isomorphism and since $X_{q}$ is fibred over $[0,1]$,

\footnotetext{
${ }^{2}$ For example, one could prove this by applying [33, Theorem C.26] to the linking algebra $\left(\begin{array}{cc}\mathcal{K}(X) & X \\ X^{*} & A\end{array}\right)$, and then take the sub-bundle of the resulting bundle of $C^{*}$-algebras consisting of sections corresponding to elements of the form $\left(\begin{array}{ll}0 & \xi \\ 0 & 0\end{array}\right)$. This is a continuous, rather than upper-semicontinous, bundle because $t \mapsto$ $\|f(t)\|$ is continuous for $f \in C([0,1], A)$, and so for $x \in X$, the map $t \mapsto\left\|x+X \cdot I_{t}\right\|^{2}=\left\|\langle x, x\rangle_{A \otimes C([0,1])}(t)\right\|$ is continuous.
} 
for any $y \in X_{q}^{t}$, we have

$$
\begin{aligned}
x y & =M_{p, q}(x \otimes y)=M_{p, q}\left(x^{\prime} \otimes f \cdot y\right) \\
& =M_{p, q}\left(\left(x^{\prime} \otimes y\right) \cdot f\right)=M_{p, q}\left(x^{\prime} \otimes y\right) \cdot f=x^{\prime} y \cdot f \in X_{p q} \cdot I_{t} .
\end{aligned}
$$

So if $x+X_{p} \cdot I_{t}=x^{\prime}+X_{p} \cdot I_{t}$ then $x y-x^{\prime} y=\left(x-x^{\prime}\right) y \in X_{p q} \cdot I_{t}$. A simple argument using associativity and distributivity of multiplication shows that if $y+X_{q} \cdot I_{t}=y^{\prime}+X_{q} \cdot I_{t}$, then for any $x \in X_{q}$ we have $x y=x y^{\prime}$, and it follows that the formula for multiplication is well-defined.

For $x, x^{\prime} \in X_{p}$ and $y, y^{\prime} \in X_{q}$, blurring, where appropriate, the distinction betwen $C([0,1], A) / I_{t}$ and $A$, we have

$$
\begin{aligned}
\left\langle x y+X_{p q} \cdot I_{t},\right. & \left.x^{\prime} y^{\prime}+X_{p q} \cdot I_{t}\right\rangle_{A} \\
& =\left\langle x y, x^{\prime} y^{\prime}\right\rangle_{C([0,1], A)}+I_{t} \\
& =\left\langle x \otimes y, x^{\prime} \otimes y^{\prime}\right\rangle_{C([0,1], A)}+I_{t} \\
& =\left\langle x \otimes y+\left(X_{p} \otimes_{C([0,1], A)} X_{q}\right) \cdot I_{t},\right. \\
& \left.\quad x^{\prime} \otimes y^{\prime}+\left(X_{p} \otimes_{C([0,1], A)} X_{q}\right) \cdot I_{t}\right\rangle_{C([0,1], A)}+I_{t} \\
& =\left\langle\left(x+X_{p} \cdot I_{t}\right) \otimes\right.
\end{aligned}
$$

So the multiplication map described above defines isomorphisms $X_{p}^{t} \otimes_{A} X_{q}^{t} \cong X_{p q}^{t}$ of Hilbert modules. Associativity follows from associativity of multiplication in $X$.

For the second-last assertion, first recall from [21, Lemma A.2] that if $T \in \mathcal{K}\left(X_{p}\right)$ is compact and $\varepsilon_{t}: C([0,1], A) \rightarrow A$, then $T \otimes_{\varepsilon_{t}} 1$ belongs to $\mathcal{K}\left(X_{p} \otimes_{\varepsilon_{t}} A\right)$ for all $t \in[0,1]$ 3 The map $x \otimes a \mapsto\left(x+X \cdot I_{t}\right) \cdot a$ is an isomorphism $X_{p} \otimes_{\varepsilon_{t}} A \cong X_{p}^{t}$ that intertwines $\phi_{p}(f) \otimes 1$ with $\phi_{p}^{t}(f(t))$ for $f \in C([0,1], A)$ and $t \in[0,1]$. So we deduce that if $\phi_{p}(f)$ is compact then each $\phi_{p}^{t}(f(t))$ is compact. Thus if $A \otimes C([0,1])$ acts by compacts on $X_{p}$, then $A$ acts by compacts on each $X_{p}^{t}$.

For the last assertion, first note that if each $\phi_{p}^{t}$ is injective, then each $\phi_{p}^{t}$ is isometric, and so $\left\|\phi_{p}(f)\right\|=\sup _{t \in[0,1]}\left\|\phi_{p}^{t}(f(t))\right\|=\sup _{t \in[0,1]}\|f(t)\|=\|f\|$. For the converse implication, suppose that $C([0,1], A)$ acts by compacts on $X_{p}$. We will first show that for any $f \in$ $C([0,1], A)$ and any $p \in P$, the map $t \mapsto\left\|\phi_{p}^{t}(f(t))\right\|$ is continuous. To this end, note that the second paragraph of the proof of [21, Lemma A.2] shows that the module $X_{p}$ is a $\mathcal{K}\left(X_{p}\right)-C([0,1], A)$-imprimitivity bimodule, and the open map $\operatorname{Prim}\left(\mathcal{K}\left(X_{p}\right)\right) \rightarrow[0,1]$ arising from the $C([0,1])$-structure as in [33, Theorem C.26] is the composition of the Rieffel homeomorphism for $X_{p}$ with the canonical map $\operatorname{Prim}(C([0,1], A)) \rightarrow[0,1]$. Since this final map is open, so is the composition, and we deduce from the final assertion of [33, Theorem C.26] that $\mathcal{K}\left(X_{p}\right)$ is a $C([0,1])$-algebra. Consequently, if $T \in \mathcal{K}\left(X_{p}\right)$, the map $t \mapsto\left\|T \otimes_{\varepsilon_{t}} 1\right\|$ is continuous. Since we are assuming that the left action of $C([0,1], A)$ on $X_{p}$ is by compacts, we see that $t \mapsto\left\|\phi_{p}^{t}(f(t))\right\|$ is continuous for each $f \in C([0,1], A)$.

Now suppose that there exists $t \in[0,1]$ such that $\phi_{p}^{t}$ is not injective. Then there exists $a \in A$ with $\|a\|=1$ such that $\phi_{p}^{t}(a)=0$. By continuity of the norm, there exists an open neighbourhood $U$ of $t$ in $[0,1]$ such that $\left\|\phi_{p}^{s}(a)\right\|<1 / 2$ for all $s \in U$. Choose $f \in C_{0}(U) \subseteq C([0,1])$ with $0 \leq f \leq 1$ and $f(t)=1$. The function $f a \in C([0,1], A)$ given

\footnotetext{
${ }^{3}$ As stated, [21, Lemma A.2] asserts that these two conditions are equivalent, but the " $\Leftarrow$ " implication is incorrect in general.
} 
by $(f a)(s)=f(s) a$ satisfies $\|f a\|=\|a\|=1$, and $\left\|\phi_{p}(f a)\right\|=\sup _{s \in[0,1]}\left\|\phi_{p}^{s}((f a)(s))\right\|=$ $\sup _{s \in U} f(s)\left\|\phi_{p}^{s}(a)\right\|<1 / 2$. So $\phi_{p}$ is not isometric and therefore not injective.

The previous Lemma enables us to adopt the following definition of a homotopy of product systems.

Definition 3.2. Let $A$ be a $C^{*}$-algebra, and let $P$ be a semigroup. Let $Y$ and $Z$ be product systems over $P$ with coefficient algebra $A$. A homotopy of product systems from $Y$ to $Z$ is a nondegenerate product system $X$ over $P$ with coefficient algebra $C([0,1], A)$ that is fibred over $[0,1]$ such that the product system $X^{0}$ is isomorphic to $Y$ and the product system $X^{1}$ is isomorphic to $Z$. We say that $Y$ and $Z$ are homotopic.

Example 3.3. Let $X$ be a product system over $P$ with coefficient algebra $A$. For each $p \in P$, define $Y_{p}:=X_{p} \otimes C([0,1])$. Define multiplication on $Y=\left\{Y_{p}\right\}_{p \in P}$ by

$$
(x \otimes f)(y \otimes g):=x y \otimes f g .
$$

We first claim that $Y$ is a homotopy of product systems from $X$ to $X$. It is easy to check that $Y$ is a nondegenerate product system over $A \otimes C([0,1])$, using that the external tensor product factors through the internal tensor product of Hilbert bimodules. It is then a homotopy of product systems by definition, and it is standard that the quotient $X_{p} \otimes C([0,1]) /\left(X_{p} \otimes C_{0}([0,1] \backslash\{t\}) \cong X_{p}\right.$, so $Y$ is a homotopy of product systems from $X$ to $X$.

We now claim that

$$
\mathcal{N} \mathcal{O}_{Y} \cong \mathcal{N} \mathcal{O}_{X} \otimes C([0,1]) \quad \text { and } \quad \mathcal{N} \mathcal{T}_{Y} \cong \mathcal{N} \mathcal{T}_{X} \otimes C([0,1])
$$

The universal property of $\mathcal{N} \mathcal{O}_{X}$ gives a homomorphism $\rho: \mathcal{N} \mathcal{O}_{X} \rightarrow \mathcal{N} \mathcal{O}_{Y}$ such that $\rho\left(j_{p}(x)\right)=j_{p}\left(x \otimes 1_{C([0,1])}\right)$ for all $p \in P$ and $x \in X$. It is routine to check that there is a homomorphim $\sigma: C([0,1]) \rightarrow \mathcal{Z} \mathcal{M}\left(\mathcal{N O}_{Y}\right)$ such that $\sigma(f) j_{p}(x \otimes g)=j_{p}(x \otimes f g)$. The universal property of the tensor product then gives a homomorphism $(\rho \otimes \pi): \mathcal{N} \mathcal{O}_{X} \otimes$ $C([0,1]) \rightarrow \mathcal{N O}_{Y}$ such that $(\rho \otimes \pi)\left(j_{p}(x) \otimes f\right)=\rho\left(j_{p}(x)\right) \sigma(f)=j_{p}(x \otimes f)$.

For each $p$ the map $x \otimes f \mapsto j_{p}(x) \otimes f$ is a module map from $Y_{p}$ to $\mathcal{N} \mathcal{O}_{X} \otimes C([0,1])$, and routine calculations show that these assemble into a representation $\tau$ of $Y$ in $\mathcal{N} \mathcal{O}_{X} \otimes$ $C([0,1])$. The modules $\widetilde{Y}_{p}$ invoked in the definition of Cuntz-Pimsner covariance are canonically isomorphic with the corresponding modules $\widetilde{X}_{p} \otimes C([0,1])$. Likewise, each $\mathcal{K}\left(X_{p} \otimes C([0,1])\right)$ is canonically isomorphic to $\mathcal{K}\left(X_{p}\right) \otimes C([0,1])$. A sum $\sum_{p \in F} \widetilde{\iota}_{p}^{s}\left(T_{p} \otimes f_{p}\right)$ is zero in $\mathcal{L}\left(\widetilde{Y}_{s}\right)$ for large $s$ if and only if each $\sum_{p \in F} \iota_{p}^{s}\left(f_{p}(t) T_{p}\right)$ is eventually zero in $\mathcal{L}\left(\widetilde{X}_{s}\right)$, and we deduce that $\tau$ is Cuntz-Nica-Pimsner covariant, and therefore induces a homomorphism $\Pi \tau: \mathcal{N} \mathcal{O}_{Y} \rightarrow \mathcal{N} \mathcal{O}_{X} \otimes C([0,1])$. It is routine to see that $\Pi \tau$ and $(\rho \otimes \pi)$ are mutually inverse, and hence isomorphisms.

Proposition 3.4. Let $A$ be a $C^{*}$-algebra and let $P$ be a semigroup. Then homotopy of product systems is an equivalence relation on the collection of product systems over $P$ of Hilbert bimodules over A.

Proof. Example 3.3 shows that the external tensor product $Y \otimes C([0,1])$ is a homotopy from $Y$ to $Y$. let $F:[0,1] \rightarrow[0,1]$ be the flip homeomorphism $F(t)=1-t$, and let $X$ be a homotopy from $Y$ to $Z$. Then there is a product system $F_{*} X$ given by setting $\left(F_{*} X\right)_{p}=X_{p}$ as a vector space, but with inner-product and actions given by 
$a: x=(a \circ F) \cdot x, x: a=x \cdot(a \circ F)$ and $\langle\langle x, y\rangle\rangle_{C([0,1], A)}=\langle x, y\rangle_{C([0,1], A)} \circ F$. This $F_{*}(X)$ is a homotopy from $Z$ to $Y$.

Finally, suppose that $W$ is a homotopy from $Y_{1}$ to $Y_{2}$, and $X$ is a homotopy from $Y_{2}$ to $Y_{3}$. Then, by definition, there are isomorphisms of product systems $U: W^{1} \rightarrow Y_{2}$ and $V: X^{0} \rightarrow Y_{2}$. Define subspaces $Z_{p} \leq W_{p} \oplus X_{p}$ by

$$
Z_{p}:=\left\{(w, x) \in W_{p} \oplus X_{p}: U\left(w+W_{p} \cdot I_{1}\right)=V\left(x+X_{p} \cdot I_{0}\right)\right\} .
$$

Routine calculations show that each $Z_{p}$ is invariant for the left and right actions of the subalgebra $B:=\{(a, b) \in C([0,1], A) \oplus C([0,1], A): a(1)=b(0)\}$, and also that $\left\langle Z_{p}, Z_{p}\right\rangle_{C([0,1], A) \oplus C([0,1], A)} \subseteq B$. It is straightforward to check that the product-system structure on $W \oplus X$ restricts to give $Z$ the structure of a nondegenerate product system over $P$ of Hilbert bimodules over $B$. We have an isomorphism $\varphi: B \rightarrow C([0,1], A)$ given by

$$
\varphi(a, b)(t)= \begin{cases}a(2 t) & \text { if } t \leq 1 / 2 \\ b(2 t-1) & \text { if } t>1 / 2 .\end{cases}
$$

Using this to view $Z$ as a product system of Hilbert bimodules over $C([0,1], A)$, we see that $Z$ is fibred over $[0,1]$ and that $Z^{0}=W^{0} \cong Y_{1}$ while $Z^{1}=X^{1} \cong Y_{3}$; so $Z$ is a homotopy from $Y_{1}$ to $Y_{3}$.

We now begin exploring the relation between $\mathcal{N} \mathcal{T}_{X}$ and $\mathcal{N} \mathcal{T}_{X^{t}}$ for a homotopy $X$ of product systems. Our goal is to show the commutativity of the following diagram.

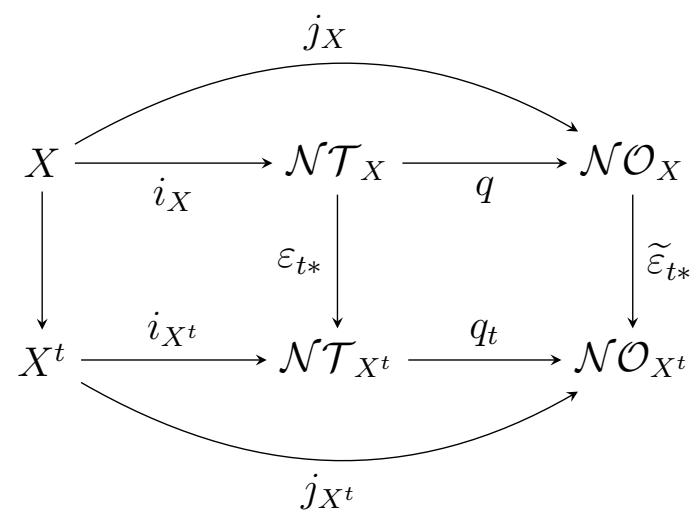

Proposition 3.5. Let $A$ be a $C^{*}$-algebra, let $P$ be a quasi-lattice ordered semigroup, and let $X$ be a nondegenerate, compactly aligned product system over $P$ of Hilbert bimodules over $C([0,1], A)$. Suppose that $X$ is fibred over $[0,1]$.

(1) There is a unique homomorphism $\iota: C([0,1]) \rightarrow \mathcal{Z} \mathcal{M}\left(\mathcal{N} \mathcal{T}_{X}\right)$ such that $\iota(f) i_{e}(g)=$ $i_{e}(t \mapsto f(t) g(t))$ for all $g \in C([0,1], A)$, and this $\iota$ is unital.

(2) For each $t \in[0,1]$, let $I_{t}^{\mathcal{T}}$ be the ideal $\{\iota(f) b: f \in C([0,1]), f(t)=0$, and $b \in$ $\left.\mathcal{N} \mathcal{T}_{X}\right\}$. There is a surjective homomorphism $\varepsilon_{t *}: \mathcal{N} \mathcal{T}_{X} \rightarrow \mathcal{N} \mathcal{T}_{X^{t}}$ such that $\varepsilon_{t *}\left(i_{p}(x)\right)=i_{p}\left(x+X_{p} \cdot I_{t}\right)$ for all $p \in P$ and $x \in X_{p}$, and we have $\operatorname{ker}\left(\varepsilon_{t *}\right)=I_{t}^{\mathcal{T}}$.

(3) Let $q: \mathcal{N} \mathcal{T}_{X} \rightarrow \mathcal{N} \mathcal{O}_{\mathcal{X}}$ and $q_{t}: \mathcal{N} \mathcal{T}_{X^{t}} \rightarrow \mathcal{N} \mathcal{O}_{X^{t}}$ be the quotient maps. Then $\jmath:=q \circ \iota$ is a unital homomorphism $\jmath: C([0,1]) \rightarrow \mathcal{Z} \mathcal{M}\left(\mathcal{N O}_{X}\right)$, each $\varepsilon_{t *}$ descends to a homormophism $\tilde{\varepsilon}_{t *}: \mathcal{N O} \mathcal{O}_{X} \rightarrow \mathcal{N} \mathcal{O}_{X^{t}}$, and if we write $I_{t}^{\mathcal{O}}:=q\left(I_{t}^{\mathcal{T}}\right)$ then we have $I_{t}^{\mathcal{O}}=\left\{\jmath(f) b: f \in C([0,1]), f(t)=0\right.$, and $\left.b \in \mathcal{N O}_{X}\right\}$.

(4) Suppose that $P$ is directed, and that for each $p \in P$ the left action of $C([0,1], A)$ on $X_{p}$ is injective and by compacts. Then $\operatorname{ker}\left(\tilde{\varepsilon}_{t *}\right)=I_{t}^{\mathcal{O}}$. 
Proof. (1) Since $X$ is nondegenerate, any approximate identity $\left(a_{i}\right)$ for $C([0,1], A)$ satisfies $a_{i} \cdot x, x \cdot a_{i} \rightarrow x$ for all $x \in X$. Hence $i_{e}\left(a_{i}\right) i_{p}(x), i_{p}(x) i_{e}\left(a_{i}\right) \rightarrow i_{p}(x)$ for all $p \in P$ and $x \in X_{p}$, and we deduce that $i_{e}: C([0,1], A) \rightarrow \mathcal{N} \mathcal{T}_{X}$ is nondegenerate, and so extends to a unital homomorphism $\tilde{i}_{e}: \mathcal{M}(C([0,1], A)) \rightarrow \mathcal{M}\left(\mathcal{N} \mathcal{T}_{X}\right)$. Since $\mathcal{M}(C([0,1], A))=$ $C([0,1], \mathcal{M}(A))$, this $\tilde{i}_{e}$ restricts to a unital homomorphism $\iota: C([0,1]) \rightarrow \mathcal{M}\left(\mathcal{N} \mathcal{T}_{X}\right)$. The range of $\iota$ is central because for each $x \in X_{p}$ we have $\iota(f) i_{p}(x)=i_{p}(f \cdot x)=i_{p}(x \cdot f)=$ $i_{p}(x) \iota(f)$ because $X_{p}$ is fibred over $[0,1]$. This homomorphism clearly satisfies $\iota(f) i_{e}(g)=$ $i_{e}(t \mapsto f(t) g(t))$, and for uniqueness, we observe that if $\iota^{\prime}: C([0,1]) \rightarrow \mathcal{Z} \mathcal{M}\left(\mathcal{N} \mathcal{T}_{X}\right)$ satisfies the same formula, then for any $x \in X$ we can use Cohen factorisation to write $x=y \cdot g$ for some $g \in C([0,1], A)$, and then $\iota^{\prime}(f) i_{p}(x)=\iota^{\prime}(f) i_{p}(y) i_{e}(g)=i_{p}(y) \iota^{\prime}(f) i_{e}(g)$ by centrality, and since $\iota^{\prime}(f) i_{e}(g)=i_{e}(f g)$ by assumption, we then have $\iota^{\prime}(f) i_{p}(x)=$ $i_{p}(y) i_{e}(f g)=i_{p}(y \cdot(f g))=i_{p}(x \cdot f)$, and the same calculation applied to $\iota$ instead of $\iota^{\prime}$ shows that this is also equal to $\iota(f) i_{p}(x)$. Since elements of the form $i_{p}(x) i_{q}(y)^{*}$ are dense in $\mathcal{N} \mathcal{T}_{X}$, it follows that $\iota=\iota^{\prime}$.

(2) We first show that $\left\{\varepsilon_{t *} \circ i_{p}\right\}_{p \in P}$ is a Nica covariant representation of $X$ in $\mathcal{N} \mathcal{T}_{X^{t}}$. It is a representation because $i$ is a representation; in particular, if $x \in X_{p}, y \in X_{q}$ we have

$$
\left(\varepsilon_{t *} \circ i_{p}(x)\right)\left(\varepsilon_{t *} \circ i_{q}(y)\right)=i_{p}\left(x+X_{p} \cdot I_{t}\right) i_{q}\left(y+X_{q} \cdot I_{t}\right)=i_{p q}\left(x y+X_{p q} \cdot I_{t}\right)=\varepsilon_{t *}\left(i_{p q}(x y)\right),
$$

and (for $x, y \in X_{p}$ )

$$
\begin{aligned}
i_{e}\left(\langle x, y\rangle_{A \otimes C([0,1])}+X_{e} \cdot I_{t}\right) & =i_{p}\left(x+X_{p} \cdot I_{t}\right)^{*} i_{p}\left(y+X_{p} \cdot I_{t}\right)=\left(\varepsilon_{t *} \circ i_{p}(x)\right)^{*}\left(\varepsilon_{t *} \circ i_{p}(y)\right) \\
& =\varepsilon_{t *} \circ i_{e}\left(\langle x, y\rangle_{A \otimes C([0,1])} .\right.
\end{aligned}
$$

To see that it is Nica covariant, first note that for $x, y \in X_{p}$, Equation (2.1) shows that $\left(\varepsilon_{t *} \circ i\right)^{(p)}=\varepsilon_{t *} \circ i^{(p)}$. Now fix $S \in \mathcal{K}\left(X_{p}\right)$ and $T \in \mathcal{K}\left(X_{q}\right)$. Then

$$
\left(\varepsilon_{t *} \circ i\right)^{(p)}(S)\left(\varepsilon_{t *} \circ i\right)^{(q)}(T)=\varepsilon_{t *}\left(i^{(p)}(S) i^{(q)}(T)\right)
$$

and $\left(\varepsilon_{t *} \circ i\right)^{(p \vee q)}\left(\iota_{p}^{p \vee q}(S) \iota_{q}^{p \vee q}(T)\right)=\varepsilon_{t *}\left(i^{(p \vee q)}\left(\iota_{p}^{p \vee q}(S) \iota_{q}^{p \vee q}(T)\right)\right)$, and so $\varepsilon_{t *} \circ i$ is Nica covariant because $i$ is.

The universal property of $\mathcal{N} \mathcal{T}_{X}$ now yields a homomorphism $\varepsilon_{t *}: \mathcal{N} \mathcal{T}_{X} \rightarrow \mathcal{N} \mathcal{T}_{X^{t}}$. This homomorphism is surjective because each $i_{p}\left(x+X_{p} \cdot I_{t}\right)=\varepsilon_{t *}\left(i_{p}(x)\right)$ and each $i_{e}(a)=$ $\varepsilon_{t *}\left(i_{e}\left(a \otimes 1_{C([0,1])}\right)\right)$ belongs to its range.

To see that $\operatorname{ker}\left(\varepsilon_{t *}\right)=I_{t}^{\mathcal{T}}$, we first observe that for any $f \in C_{0}([0,1] \backslash\{t\}), x \in X_{p}$,

$$
\varepsilon_{t *}\left(\iota(f) i_{p}(x)\right)=\varepsilon_{t *}\left(i_{p}\left(\left(f \otimes 1_{A}\right) \cdot x\right)=i_{p}\left(x \cdot\left(f \otimes 1_{A}\right)+X_{p} \cdot I_{t}\right)=0,\right.
$$

since $x \cdot\left(f \otimes 1_{A}\right) \in X_{p} \cdot I_{t}$. Consequently, $I_{t}^{\mathcal{T}} \subseteq \operatorname{ker} \varepsilon_{t *}$. To establish the other containment, we will construct a Nica-covariant representation $\psi$ of $X^{t}$ in $\mathcal{N} \mathcal{T}_{X} / I_{t}^{\mathcal{T}}$, such that the resulting homomorphism $\Pi \psi: \mathcal{N} \mathcal{T}_{X^{t}} \rightarrow \mathcal{N} \mathcal{T}_{X} / I_{t}^{\mathcal{T}}$ satisfies $\varepsilon_{t *} \circ \Pi \psi=$ id. We aim to define

$$
\psi\left(i_{p}\left(x+X_{p} \cdot I_{t}\right)\right)=i_{p}(x)+I_{t}^{\mathcal{T}}
$$

To see that this is well defined, observe that for all $x, y \in X_{p}$, we have

$$
\begin{aligned}
\left(i_{p}(x)+I_{t}^{\mathcal{T}}\right)^{*}\left(i_{p}(y)+I_{t}^{\mathcal{T}}\right) & =i_{p}(x)^{*} i_{p}(y)+I_{t}^{\mathcal{T}}=i_{e}\left(\langle x, y\rangle_{A \otimes C([0,1])}\right)+I_{t}^{\mathcal{T}} \\
& =\left\langle i_{p}\left(x+X_{p} \cdot I_{t}\right), i_{p}\left(y+X_{p} \cdot I_{t}\right)\right\rangle_{A} .
\end{aligned}
$$

Hence the formula for $\psi$ determines an isometric map from $X_{p}^{t}$ to $\mathcal{N} \mathcal{T}_{X} / I_{t}^{\mathcal{T}}$. An argument like that given for $\varepsilon_{t *} \circ i$ above shows that $\psi$ is a Nica-covariant representation. To see 
that $\varepsilon_{t *} \circ \psi=\mathrm{id}$, we compute:

$$
\varepsilon_{t *}\left(\psi\left(i_{p}\left(x+X_{p} \cdot I_{t}\right)\right)=\varepsilon_{t *}\left(i_{p}(x)+I_{t}^{\mathcal{T}}\right)=\varepsilon_{t *}\left(i_{p}(x)\right)=i_{p}\left(x+X_{p} \cdot I_{t}\right) .\right.
$$

(3) To see that $\varepsilon_{t *}$ descends to a homomorphism $\tilde{\varepsilon}_{t *}: \mathcal{N} \mathcal{O}_{X} \rightarrow \mathcal{N} \mathcal{O}_{X^{t}}$, it suffices to show that if $q_{t}: \mathcal{N} \mathcal{T}_{X^{t}} \rightarrow \mathcal{N} \mathcal{O}_{X^{t}}$ is the quotient map, then $q_{t} \circ \varepsilon_{t *} \circ i_{p}$ is a Cuntz-Pimsner covariant representation of $X$. Since the actions of $A \otimes C([0,1])$ on the modules $X_{p}$ are implemented by injective homomorphisms, the modules $\tilde{X}_{p}$ of 32 are just the original modules $X_{p}$, and the homomorphisms $\tilde{\iota}_{p}^{p q}: \mathcal{L}\left(X_{p}\right) \rightarrow \mathcal{L}\left(\widetilde{X}_{p q}\right)$ are the standard inclusions $\iota_{p}^{p q}: \mathcal{L}\left(X_{p}\right) \rightarrow \mathcal{L}\left(X_{p q}\right)$ characterised by $\iota_{p}^{p q}(S)(x y)=(S x) y$ for $x \in X_{p}$ and $y \in X_{q}$. Write $\iota[t]_{p}^{p q}: \mathcal{L}\left(X_{p}^{t}\right) \rightarrow \mathcal{L}\left(X_{p q}^{t}\right)$ for the corresponding inclusions for the product system $X^{t}$; and as usual for $T \in \mathcal{L}\left(X_{p}\right)$ write $T_{t}$ for the map in $\mathcal{L}\left(X_{p}^{t}\right)$ given by $T_{t}\left(x+X \cdot I_{t}\right)=T x+X \cdot I_{t}$. A routine calculation shows that for $T \in \mathcal{L}\left(X_{p}\right)$ we have $\iota[t]_{p}^{p q}\left(T_{t}\right)=\iota_{p}^{p q}(T)_{t}$. So if $F \subseteq P$ is finite and $T_{p} \in \mathcal{K}\left(X_{p}\right)$ for each $p \in F$, then

$$
\left(\sum_{p \in F} \iota_{p}^{s}\left(T_{p}\right)\right)_{t}=\sum_{p \in F} \iota[t]_{p}^{s}\left(\left(T_{p}\right)_{t}\right) \quad \text { for every } s \geq p \text { in } P .
$$

Assume $\sum_{p \in F} \iota_{p}^{s}\left(T_{p}\right)=0$ for large $s$; we will show that $\sum_{p \in F}\left(q_{t} \circ \varepsilon_{t *} \circ i\right)^{(p)}\left(T_{p}\right)=0$. From (3.1) we see that $\sum_{p \in F} \iota[t]_{p}^{s}\left(\left(T_{p}\right)_{t}\right)=0$ for large $s$, and consequently

$$
q_{t}\left(\sum_{p \in F} i_{t}^{(p)}\left(\left(T_{p}\right)_{t}\right)\right)=q_{t}\left(\sum_{p \in F} \iota_{p}^{s}[t]\left(\left(T_{p}\right)_{t}\right)\right)=0 .
$$

Equation 2.1 shows that $\left(q_{t} \circ \varepsilon_{t *} \circ i\right)^{(p)}=\left(q_{t} \circ \varepsilon_{t *}\right) \circ i^{(p)}$. Hence

$$
\sum_{p \in F}\left(q_{t} \circ \varepsilon_{t *} \circ i\right)^{(p)}\left(\left(T_{p}\right)_{t}\right)=\sum_{p \in F}\left(q_{t} \circ \varepsilon_{t *}\right) \circ i^{(p)}\left(T_{p}\right)=0 .
$$

That $I_{t}^{\mathcal{O}}=\left\{\jmath(f) b: f \in C([0,1]), f(t)=0\right.$, and $\left.b \in \mathcal{N O}_{X}\right\}$ follows from the definition of $I_{t}^{\mathcal{T}}$.

(4) Since $I_{t}^{\mathcal{O}}=q\left(I_{t}^{\mathcal{T}}\right)=q\left(\operatorname{ker}\left(\varepsilon_{t *}\right)\right)$, we clearly have $I_{t}^{\mathcal{O}} \subseteq \operatorname{ker}\left(\tilde{\varepsilon}_{t *}\right)$. To see that $\operatorname{ker}\left(\tilde{\varepsilon}_{t *}\right)=$ $q\left(I_{t}^{\mathcal{T}}\right)$, it suffices to show that if $q_{t}: \mathcal{N} \mathcal{T}_{X} / I_{t}^{\mathcal{T}} \rightarrow \mathcal{N} \mathcal{O}_{X} / I_{t}^{\mathcal{O}}$ is the quotient map, then the Nica-covariant representation $\psi: X^{t} \rightarrow \mathcal{N} \mathcal{T}_{X} / I_{t}^{\mathcal{T}}$ defined above has the property that $q_{t} \circ$ $\psi$ is Cuntz-Nica-Pimsner covariant. For each $p \in P$, let $\phi_{p}^{t}: A \rightarrow \mathcal{K}\left(X_{p}^{t}\right)$ be the injective homomorphism that implements the left action; and let $\phi_{p}: A \otimes C([0,1]) \rightarrow \mathcal{K}\left(X_{p}\right)$ be the corresponding map for $X$. We invoke [32, Proposition 5.1(2)], which says that it suffices to show that for each $a \in A$ and each $p \in P$, we have $\left(q_{t} \circ \psi\right)^{(p)}\left(\phi_{p}^{t}(a)\right)-\left(q_{t} \circ \psi_{e}\right)(a)=0$. By [32, Proposition 5.1(2)], we have $i^{(p)}\left(\phi_{p}\left(a \otimes 1_{[0,1]}\right)\right)-i_{e}\left(a \otimes 1_{[0,1]}\right) \in \operatorname{ker}(q)$. Since the identification of $A \otimes C([0,1])) / A \otimes C_{0}([0,1] \backslash\{t\})$ with $A$ carries $a \otimes 1_{[0,1]}$ to $a$, we have

$$
\begin{aligned}
\left(q_{t} \circ \psi\right)^{(p)}\left(\phi_{p}^{t}(a)\right)-\left(q_{t} \circ \psi_{e}\right)(a) & =q_{t}\left(\psi^{(p)}\left(\phi_{p}^{t}(a)\right)-\psi_{e}(a)\right) \\
& =q_{t}\left(i^{(p)}\left(\phi_{p}\left(a \otimes 1_{[0,1]}\right)\right)-i_{e}\left(a \otimes 1_{[0,1]}\right)+I_{t}^{\mathcal{T}}\right) \\
& =q\left(i^{(p)}\left(\phi_{p}\left(a \otimes 1_{[0,1]}\right)\right)-i_{e}\left(a \otimes 1_{[0,1]}\right)\right)+I_{t}^{\mathcal{O}}=0 .
\end{aligned}
$$

Remark 3.6. It is unclear whether the hypothesis that $p \vee q<\infty$ for all $p, q \in P$ is necessary for the final assertion of Proposition 3.5)(3). The issue is that to establish the final assertion without this hypothesis, we would need to verify the Cuntz-Pimsner relation for the map $q \circ \psi$ in the final paragraph of the proof. That is, given a finite $F \subseteq P$ and elements $T_{p}^{t} \in \mathcal{K}\left(X_{p}^{t}\right)$ such that $\sum_{p} \iota[t]_{p}^{s}\left(T_{p}^{t}\right)=0$ for large $s$, we would need to show 
that we could find representatives $T_{p} \in \mathcal{K}\left(X_{p}\right)$ of the operators $T_{p}^{t}$ with the property that $\sum_{p} \iota_{p}^{s}\left(T_{p}\right)=0$ for large $s$; it is not clear to us whether this is so.

Our main theorem states that homotopy of product systems over $\mathbb{N}^{k}$ preserves the $K$-theory of their Cuntz-Nica-Pimsner algebras.

Theorem 3.7. Let $X$ be a homotopy of product systems over $\mathbb{N}^{k}$ whose coefficient algebra $A \otimes C([0,1])$ acts faithfully by compact operators. For any $t \in[0,1]$, the evaluation map $\varepsilon_{t}$ induces an isomorphism

$$
K_{*}\left(\mathcal{N} \mathcal{O}_{X^{t}}\right) \cong K_{*}\left(\mathcal{N O} \mathcal{O}_{X}\right)
$$

In particular, if $A$ satisfies the UCT and each $X_{n}$ is countably generated, then each $\mathcal{N O}_{X^{t}}$ is $K K$-equivalent to $\mathcal{N O}_{X}$.

Proof. We will proceed by induction on $k$. Recall that $X_{0} \cong C([0,1], A)$, and so $X_{0}^{t} \cong A$. Hence, if $k=0$ then $\mathcal{N} \mathcal{O}_{X}=C([0,1], A)$ and $\mathcal{N} \mathcal{O}_{X^{t}}=A$, and $\varepsilon_{t *}$ is just the evaluation map $\varepsilon_{t}: C([0,1], A) \rightarrow A$. Thus $\varepsilon_{t *}$ induces a $K K$-equivalence.

Now suppose that the result holds for all product systems over $\mathbb{N}^{k-1}$. Let $X_{<k}$ be the product system over $\mathbb{N}^{k-1}$ obtained by restriction of $X$, which is also a homotopy of product systems with coefficient algebra $A$. Theorem 4.7 of [6] (see also [5, Theorem 3.4.21]) shows that there is a product system $Y$ of Hilbert $\mathcal{N} \mathcal{O}_{X_{<k}}$-bimodules over $\mathbb{N}$ in which the left action is faithful and by compact operators and such that $\mathcal{N} \mathcal{O}_{X} \cong \mathcal{N O}_{Y}$. As $Y$ is a product system over $\mathbb{N}, \mathcal{N} \mathcal{O}_{Y}=\mathcal{O}_{Y_{1}}$.

By definition (see [6, Proposition 4.3] or [5, Proposition 3.4.7]), if $\jmath: \mathcal{N O}_{X_{<k}} \rightarrow \mathcal{N} \mathcal{O}_{X}$ is the homomorphism induced by the inclusion $X_{<k} \hookrightarrow X$, then the module $Y_{1}$ is equal to $\overline{\operatorname{span}}\left\{i_{e_{k}}(\xi) \jmath(b): \xi \in X_{e_{k}}, b \in \mathcal{N O}_{X_{<k}}\right\} \subseteq \mathcal{N} \mathcal{O}_{X}$, and similarly for $Y_{1}^{t}$ for each fixed $t$. So the map $\varepsilon_{t *}: \mathcal{N} \mathcal{O}_{X} \rightarrow \mathcal{N} \mathcal{O}_{X^{t}}$ restricts to a bimodule map $\left(\varepsilon_{t *}^{Y}, \varepsilon_{t *}^{<k}\right):\left(Y_{1}, \mathcal{N} \mathcal{O}_{X_{<k}}\right) \rightarrow$ $\left(Y_{1}^{t}, \mathcal{N O}_{X_{<k}^{t}}\right)$, and by definition the homomorphism $\mathcal{N O}_{X} \rightarrow \mathcal{N} \mathcal{O}_{X^{t}}$ induced by this bimodule map is the original $\varepsilon_{t *}$. Hence the final statement (concerning naturality) of [21, Theorem 4.4], shows that Pimsner's six-term exact sequences in $K K$-theory [24, Theorem 4.9(1)] applied with $B=\mathbb{C}$ to each of $Y_{1}$ and $Y_{1}^{t}$, assemble into the following commuting diagram:

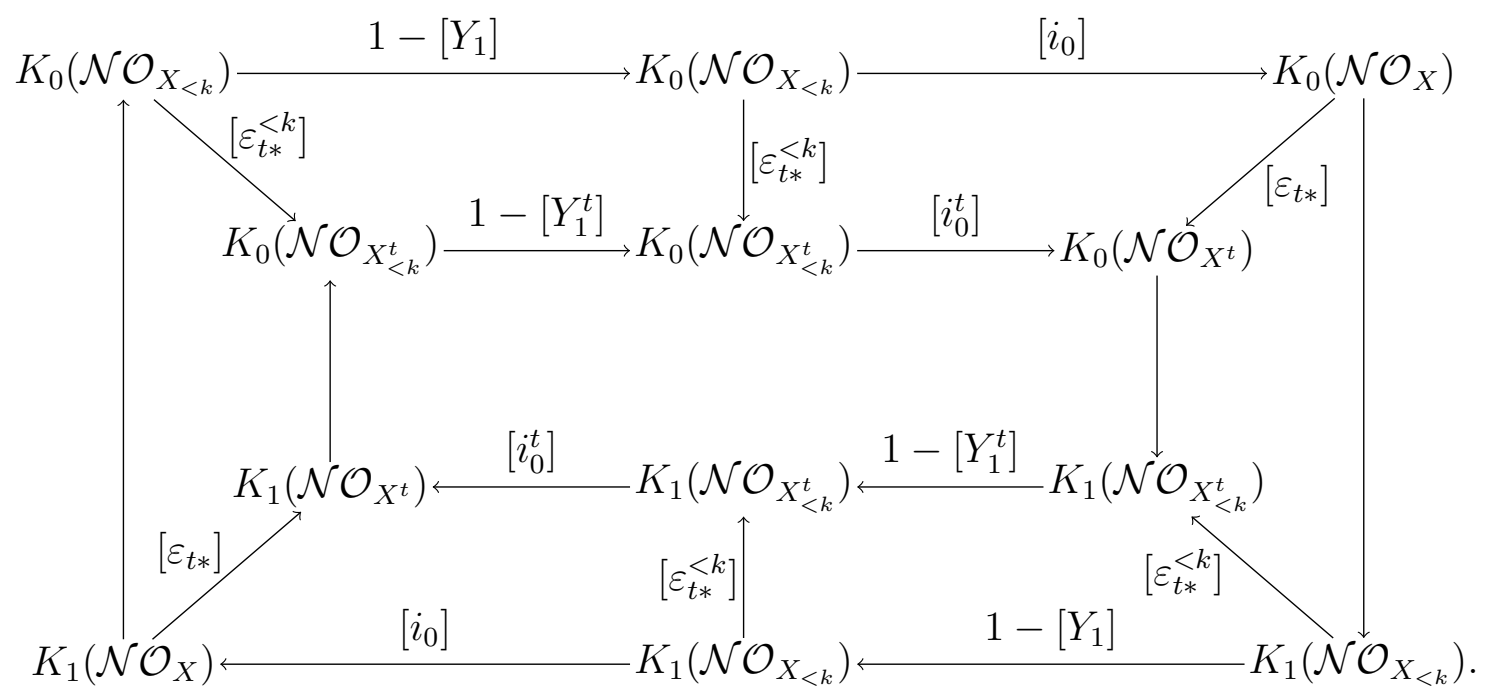


The inductive hypothesis implies that the upper-left, upper-middle, lower-right and lower-middle outside-to-inside maps are isomorphisms, and so the Five Lemma shows that the remaining two outside-to-inside maps are isomorphisms as well.

Corollary 5.21 of [6] establishes that if $A$ satisfies the Universal Coefficient Theorem, then our hypotheses on $A$ and $X$ imply that $\mathcal{N O}_{X}$ and $\mathcal{N} \mathcal{O}_{X^{t}}$ also satisfy the UCT. Consequently, the above isomorphisms in $K$-theory give a $K K$-equivalence in this case.

To finish the section, we prove that the evaluation maps $\varepsilon_{t}$ for a homotopy of product systems over $\mathbb{N}^{k}$ induce $K K$-equivalences of Nica-Toeplitz algebras.

Corollary 3.8. Let $X$ be a homotopy of product systems over $\mathbb{N}^{k}$ with coefficient algebra $A$. Suppose that $A$ is $\sigma$-unital and each $X_{n}$ is countably generated. For any $t \in[0,1]$, the class of the homomorphism $\varepsilon_{t *}$ of Proposition 3.5(2) is a $K K$-equivalence from $\mathcal{N}^{\mathcal{T}_{X}}$ to $\mathcal{N} \mathcal{T}_{X^{t}}$, and in particular defines an isomorphism

$$
K_{*}\left(\mathcal{N} \mathcal{T}_{X}\right) \cong K_{*}\left(\mathcal{N} \mathcal{T}_{X^{t}}\right)
$$

Proof. By [6, Corollary 4.18], the classes of $i: C([0,1], A) \rightarrow \mathcal{N} \mathcal{T}_{X}$ and $i^{t}: A \rightarrow \mathcal{N} \mathcal{T}_{X^{t}}$ are $K K$-equivalences. Since [0,1] is contractible, the evaluation map $\varepsilon_{t}^{0}: C([0,1], A) \rightarrow A$ also induces a $K K$-equivalence. We have $i^{t} \circ \varepsilon_{t}^{0}=\varepsilon_{t *} \circ i$, and so in $K K$-theory, we have

$$
\left[\varepsilon_{t *}\right]=\left[i^{t}\right] \widehat{\otimes}\left[\varepsilon_{t}^{0}\right] \widehat{\otimes}[i]^{-1} \text {. }
$$

Since the three factors on the right-hand side are $K K$-equivalences, we deduce that $\left[\varepsilon_{t *}\right]$ is a $K K$-equivalence as well.

\section{Applications to $k$-GRAphs}

The main result of this section, Theorem 4.1, uses Theorem 3.7 to show that any two twisted Cuntz-Krieger algebras of 2-graphs with isomorphic skeletons have isomorphic $K$-groups. Our approach relies on casting the notion of homotopy of 2-cocycles for $k$ graphs (previously studied in relation to $K$-theory in $[20,10]$ ) in the language of product systems. Most of the section is aimed at proving this result about 2-graphs, but we also pause to investigate some potential applications to $k$-graphs for $k \geq 2$. Consequently, many of the results that contribute to the proof of Theorem 4.1 are stated for arbitrary $k$.

We begin by introducing the notation and definitions needed for our construction of the product systems associated to twisted $k$-graph $C^{*}$-algebras.

4.1. Background and notation for twisted $k$-graph $C^{*}$-algebras. We write $\mathbb{N}^{k}$ for the monoid of $k$-tuples of nonnegative integers under coordinatewise addition, and sometimes regard it as a small category with one object. We write $\left\{e_{1}, \ldots, e_{k}\right\}$ for the canonical generators of $\mathbb{N}^{k}$. Recall from [15] that a $k$-graph, or a higher-rank graph of rank $k$, is a countable small category $\Lambda$ equipped with a functor $d: \Lambda \rightarrow \mathbb{N}^{k}$ that satisfies the factorisation property: whenever $d(\lambda)=m+n$ there exist unique $\mu \in d^{-1}(m)$ and $\nu \in d^{-1}(n)$ such that $\lambda=\mu \nu$. We write $\Lambda^{n}:=d^{-1}(n)$. The factorisation property guarantees that $\Lambda^{0}=\left\{\operatorname{id}_{o}: o \in \operatorname{Obj}(\Lambda)\right\}$. Given subsets $E, F \subseteq \Lambda$, we write $E F=\{\mu \nu: \mu \in E, \nu \in F, s(\mu)=r(\nu)\}$. In the instance of singleton sets, we simplify notation by writing $\mu F$ rather than $\{\mu\} F$ and $E \nu$ rather than $E\{\nu\}$. 
We say that the $k$-graph $\Lambda$ is row finite with no sources if $0<\left|v \Lambda^{n}\right|<\infty$ for all $v \in \Lambda^{0}$ and $n \in \mathbb{N}^{k}$. This hypothesis, which we will assume throughout this paper, facilitates the construction and analysis of the $C^{*}$-algebra associated to the $k$-graph.

As introduced in [18, 19, there are two types of 2-cocycle on a $k$-graph $\Lambda$ : categorical and cubical. A categorical cocycle is a function $c:\{(\lambda, \mu) \in \Lambda \times \Lambda: s(\lambda)=r(\mu)\} \rightarrow \mathbb{T}$ such that $c(\lambda, \mu) c(\lambda \mu, \nu)=c(\mu, \nu) c(\lambda, \mu \nu)$ for all composable triples $(\lambda, \mu, \nu)$, and such that $c(r(\lambda), \lambda)=1=c(\lambda, s(\lambda))$ for all $\lambda$. To describe cubical cocycles, observe that if $1 \leq i_{1}<i_{2}<i_{3} \leq k$, and if $d(\lambda)=e_{i_{1}}+e_{i_{2}}+e_{i_{3}}$, then the factorisation property shows that for each $j \in\{1,2,3\}$ there are factorisations $\lambda=F_{j}^{0}(\lambda) \beta_{j}=\alpha_{j} F_{j}^{1}(\lambda)$ such that $d\left(\alpha_{j}\right)=d\left(\beta_{j}\right)=e_{i_{j}}$. A cubical 2-cocycle on a $k$-graph $\Lambda$ is a function $\phi: \bigsqcup_{i \neq j} \Lambda^{e_{i}+e_{j}} \rightarrow \mathbb{T}$ that satisfies the following cocycle identity: whenever $1 \leq i_{1}<i_{2}<i_{3} \leq k$ and $d(\lambda)=$ $e_{i_{1}}+e_{i_{2}}+e_{i_{3}}$ as above, we have

$$
\phi\left(F_{1}^{0}(\lambda)\right) \phi\left(F_{2}^{1}(\lambda)\right) \phi\left(F_{3}^{0}(\lambda)\right)=\phi\left(F_{1}^{1}(\lambda)\right) \phi\left(F_{2}^{0}(\lambda)\right) \phi\left(F_{3}^{1}(\lambda)\right) .
$$

Intuitively, if we evaluate a 2-cocycle on the faces of a 3-cube with the edges of degree $e_{i_{1}}$ coming out of the page, the edges of degree $e_{i_{2}}$ pointing vertically down, and the edges of degree $e_{i_{3}}$ pointing horizontally left, the product of the cocycle values on the front, top and left sides of the cube should be the same as the product of the cocycle values on the back, bottom, and right sides.

If $\phi$ is a cubical 2-cocycle on a row-finite $k$-graph $\Lambda$ with no sources, then a CuntzKrieger $\phi$-representation of $\Lambda$ in a $C^{*}$-algebra consists of mutually orthogonal projections $\left\{p_{v}: v \in \Lambda^{0}\right\}$ and partial isometries $\left\{s_{\mu}: \mu \in \bigsqcup_{i} \Lambda^{e_{i}}\right\}$ satisfying

(1) $s_{\mu}^{*} s_{\mu}=p_{s(\mu)}$ for all $\mu \in \bigsqcup_{i} \Lambda^{e_{i}}$,

(2) $s_{\mu^{\prime}} s_{\nu^{\prime}}=\phi(\mu \nu) s_{\mu} s_{\nu}$ whenever $1 \leq i<j \leq k$ and $\mu, \mu^{\prime} \in \Lambda^{e_{i}}$ and $\nu, \nu^{\prime} \in \Lambda^{e_{j}}$ satisfy $\mu \nu=\nu^{\prime} \mu^{\prime}$, and

(3) $p_{v}=\sum_{\mu \in v \Lambda^{e_{i}}} s_{\mu} s_{\mu}^{*}$ for all $v \in \Lambda^{0}$ and $i \leq k$.

The twisted $C^{*}$-algebra $C_{\phi}^{*}(\Lambda)$ is the universal $C^{*}$-algebra generated by a Cuntz-Krieger $\phi$-representation of $\Lambda$.

If $c$ is a categorical 2-cocycle on $\Lambda$, then the twisted $C^{*}$-algebra $C^{*}(\Lambda, c)$ is the universal $C^{*}$-algebra generated by elements $\left\{s_{\lambda}: \lambda \in \Lambda\right\}$ such that $\left\{s_{v}: v \in \Lambda^{0}\right\}$ is a family of mutually orthogonal projections, $s_{\lambda}^{*} s_{\lambda}=s_{s(\lambda)}$ for all $\lambda \in \Lambda, s_{\mu} s_{\nu}=c(\mu, \nu) s_{\mu \nu}$ whenever $s(\mu)=r(\nu)$, and $s_{v}=\sum_{\lambda \in v \Lambda^{n}} s_{\lambda} s_{\lambda}^{*}$ for all $v \in \Lambda^{0}$ and $n \in \mathbb{N}^{k}$. Theorem 4.15 and Proposition 5.7 of [19] (see also [11]) imply that for every categorical cocycle $c$ there is a cubical cocycle $\phi$ such that $C_{\phi}^{*}(\Lambda)$ is canonically isomorphic to $C^{*}(\Lambda, c)$, and conversely.

The following notions, while not standard in the literature on $k$-graphs, underlie our results in this section. Given directed graphs $E, F$ with common vertex set $E^{0}=F^{0}$, and given $v, w \in E^{0}$, we write

$$
E^{1} F^{1}:=\left\{e f: e \in E^{1}, f \in F^{1}, \text { and } s(e)=r(f)\right\},
$$

and for $v, w \in E^{0}$ we write

$$
v E^{1} F^{1} w=\left\{e f \in E^{1} F^{1}: r(e)=v \text { and } s(f)=w\right\} .
$$

By a $k$-skeleton, we mean a tuple $E=\left(E_{1}, \ldots, E_{k}\right)$ of row-finite directed graphs with no sources and with common vertex set $E^{0}$ such that for all $v, w \in E^{0}$ and all $i \neq j \leq k$, we have

$$
\left|v E_{i}^{1} E_{j}^{1} w\right|=\left|v E_{j}^{1} E_{i}^{1} w\right|
$$


Every $k$-graph $\Lambda$ gives rise to a $k$-skeleton (namely $E_{i}=\Lambda^{e_{i}}$ ). The converse is not true; however, the additional structure needed for a $k$-skeleton to give rise to a $k$-graph is described in [12].

4.2. Unitary cocycles and higher-rank graphs. The main result of this section is the following.

Theorem 4.1. Suppose that $\Lambda$ and $\Gamma$ are row-finite 2-graphs with no sources, and suppose that there exist bijections $\rho^{0}: \Lambda^{0} \rightarrow \Gamma^{0}, \rho^{e_{1}}: \Lambda^{e_{1}} \rightarrow \Gamma^{e_{1}}$ and $\rho^{e_{2}}: \Lambda^{e_{2}} \rightarrow \Gamma^{2_{e}}$ such that $\rho^{0} \circ s=s \circ \rho^{e_{i}}$ and $\rho^{0} \circ r=r \circ \rho^{e_{i}}$ for $i=1,2$. Suppose that $\phi: \Lambda^{(1,1)} \rightarrow \mathbb{T}$ and $\psi: \Gamma^{(1,1)} \rightarrow \mathbb{T}$ are cubical 2-cocycles. Then $K_{*}\left(C_{\phi}^{*}(\Lambda)\right) \cong K_{*}\left(C_{\psi}^{*}(\Gamma)\right)$.

Before proving the theorem, we state explicitly the following immediate corollary.

Corollary 4.2. Suppose that $\Lambda$ and $\Gamma$ are row-finite 2-graphs with no sources and isomorphic skeletons as in Theorem 4.1, and that $c: \Lambda^{(2)} \rightarrow \mathbb{T}$ and $c^{\prime}: \Gamma^{(2)} \rightarrow \mathbb{T}$ are categorical 2-cocycles. Then $K_{*}\left(C^{*}(\Lambda, c)\right) \cong K_{*}\left(C^{*}\left(\Gamma, c^{\prime}\right)\right)$.

Proof. By [19, Theorem 4.15 and Proposition 5.7], there exist cubical cocycles $\phi$ on $\Lambda$ and $\psi$ on $\Gamma$ such that $C^{*}(\Lambda, c) \cong C_{\phi}^{*}(\Lambda)$ and $C^{*}\left(\Gamma, c^{\prime}\right) \cong C_{\psi}^{*}(\Gamma)$. The result then follows from Theorem 4.1 .

The next lemma underlies Definition 4.4 below.

Lemma 4.3. Let $E=\left(E_{1}, E_{2}\right)$ be a 2-skeleton. For each $v, w \in E^{0}$ suppose that $U(v, w)$ is a unitary operator $U(v, w) \in \mathcal{U}\left(\mathbb{C}^{v E_{2}^{1} E_{1}^{1} w}, \mathbb{C}^{v E_{1}^{1} E_{2}^{1} w}\right)$. Let $A:=C_{0}\left(E^{0}\right)$, and for $i=1,2$, let $X_{i}:=X\left(E_{i}\right)$ be the graph bimodule of $E_{i}$ as in Example 2.1. Then there is an isomorphism $U: X_{2} \otimes_{A} X_{1} \rightarrow X_{1} \otimes_{A} X_{2}$ such that $U\left(\delta_{f} \otimes \delta_{e}\right)=U(r(f), s(e))\left(\delta_{f} \otimes \delta_{e}\right)$ for all fe $\in E_{2}^{1} E_{2}^{1}$.

Proof. Let $X_{12}$ and $X_{21}$ be the graph modules for the directed graphs $\left(E^{0}, E_{1}^{1} E_{2}^{1}, r, s\right)$ and $\left(E^{0}, E_{2}^{1} E_{1}^{1}, r, s\right)$ respectively. Routine calculations with inner products (see the proof of [26, Proposition 3.2]) show that $X_{i j} \cong X_{i} \otimes_{A} X_{j}$ via a map satisfying $\delta_{e f} \mapsto \delta_{e} \otimes \delta_{f}$, so it suffices to show that there is an isomorphism $X_{21} \rightarrow X_{12}$ satisfying $\delta_{f e} \mapsto U(r(f), s(e)) \delta_{f e}$ for all $f e \in E_{2}^{1} E_{1}^{1}$. For this, fix $f e, h g \in E_{2}^{1} E_{1}^{1}$. For $w \in E^{0}$, we calculate:

$$
\begin{aligned}
\left\langle U(r(f), s(e)) \delta_{f e}, U(r(h), s(g)) \delta_{h g}\right\rangle_{A}(w) & \\
= & \sum_{\beta \in E_{1}^{1} E_{1}^{2} w} \overline{\left(U(r(f), s(e)) \delta_{f e}\right)(\beta)}\left(U(r(h), s(g)) \delta_{h g}\right)(\beta) .
\end{aligned}
$$

This is equal to zero unless $r(h)=r(f)$ and $s(g)=s(e)$, in which case, $U(r(h), s(g))=$ $U(r(f), s(e))$, and since this is a unitary operator, we may continue the calculation

$$
\begin{aligned}
& =\delta_{r(f), r(h)} \delta_{s(e), s(g)}\left\langle U(r(f), s(e)) \delta_{f e}, U(r(f), s(e)) \delta_{h g}\right\rangle_{A}(w) \\
& =\left\langle\delta_{f e}, \delta_{h g}\right\rangle_{A}(w) .
\end{aligned}
$$

It follows by linearity and continuity that there is an inner-product-preserving linear operator

$$
U: X_{21} \rightarrow X_{12}
$$

such that $\left.U\right|_{\delta_{v} \cdot X_{21} \cdot \delta_{w}}=U(v, w)$ for all $v, w$. Since each $\left.U\right|_{\delta_{v} \cdot X_{21} \cdot \delta_{w}}$ is surjective onto $\delta_{v}$. $X_{12} \cdot \delta_{w}$, and since these subspaces span $X_{12}$, we see that $U$ is surjective and hence an isomorphism. 
Definition 4.4. Let $E=\left(E_{1}, \ldots, E_{k}\right)$ be a $k$-skeleton and $A=C_{0}\left(E_{0}\right)$. By a unitary cocycle for $E$, we mean a collection $\left\{U_{i, j}(v, w): v, w \in E^{0}, 1 \leq i<j \leq k\right\}$ of unitary operators $U_{i, j}(v, w) \in \mathcal{U}\left(\mathbb{C}^{v E_{j}^{1} E_{i}^{1} w}, \mathbb{C}^{v E_{i}^{1} E_{j}^{1} w}\right)$ such that the isomorphisms $U_{i, j}: X\left(E_{j}\right) \otimes_{A}$ $X\left(E_{i}\right) \rightarrow X\left(E_{i}\right) \otimes_{A} X\left(E_{j}\right)$ for $i<j$ given by Lemma 4.3 satisfy the cocycle identity

$$
\begin{aligned}
& \left(U_{i, j} \otimes 1_{X_{l}}\right)\left(1_{X_{j}} \otimes U_{i, l}\right)\left(U_{j, l} \otimes 1_{X_{i}}\right) \\
& \quad=\left(1_{X_{i}} \otimes U_{j, l}\right)\left(U_{i, l} \otimes 1_{X_{j}}\right)\left(1_{X_{l}} \otimes U_{i, j}\right) \quad \text { for all } 1 \leq i<j<l \leq k .
\end{aligned}
$$

Remark 4.5. If $k=2$ then the condition (4.2) is vacuous because the inequality $1 \leq$ $i<j<l \leq 2$ has no solutions. So a unitary cocycle for a 2-skeleton $\left(E_{1}, E_{2}\right)$ is nothing more than a collection $\left\{U_{1,2}(v, w): v, w \in E^{0}\right\}$ of unitary operators $U_{1,2}(v, w) \in$ $\mathcal{U}\left(\mathbb{C}^{v E_{2}^{1} E_{1}^{1} w}, \mathbb{C}^{v E_{1}^{1} E_{2}^{1} w}\right)$. In particular, since each $\left|v E_{2}^{1} E_{1}^{1} w\right|=\left|v E_{1}^{1} E_{2}^{1} w\right|$, every 2-skeleton admits many unitary cocycles.

By contrast, [17, Example 5.15(ii)] presents an example, due to Jack Spielberg, of a 3 -skeleton that cannot be the skeleton of a 3-graph, and it is straightforward to extend the arguments used in that example to see that this 3-skeleton does not admit any unitary cocycles. In particular, for $k \geq 3$ the existence of a unitary cocycle for $\left(E_{1}, \ldots, E_{k}\right)$ is a nontrivial additional hypothesis in our results henceforth. However, Proposition 4.7 below shows that there are plenty of $k$-skeletons that do admit plenty of unitary cocycles.

We are interested in unitary cocycles because they correspond exactly with product systems over $\mathbb{N}^{k}$ with generating fibres $X_{e_{i}}=X\left(E_{i}\right)$. To be precise, the results of [9] imply that every unitary cocycle determines a product system. This is the content of the next lemma.

Lemma 4.6. Let $E=\left(E_{1}, \ldots, E_{k}\right)$ be a k-skeleton, and let $A=C_{0}\left(E^{0}\right)$.

(1) Let $U=\left\{U_{i, j}(v, w): v, w \in E^{0}, 1 \leq i<j \leq k\right\}$ be a unitary cocycle for $E$. There is a unique product system $X_{U}$ over $\mathbb{N}^{k}$ with coefficient algebra $A$ such that: $X_{e_{i}}=X\left(E_{i}\right)$ for $i \leq k$; for all $1 \leq i<j \leq k$, we have $X_{e_{i}+e_{j}}=X_{e_{i}} \otimes_{A} X_{e_{j}}$; and the multiplication in $X$ satisfies, for $1 \leq i<j \leq k, \xi \in X_{e_{i}}$ and $\eta \in X_{e_{j}}$,

$$
\xi \eta=\xi \otimes \eta \quad \text { and } \quad \eta \xi=U_{i, j}(\eta \otimes \xi) .
$$

(2) Suppose that $X$ is a product system over $\mathbb{N}^{k}$ with coefficient algebra $A$ such that $X_{e_{i}} \cong X\left(E_{i}\right)$ for all $i \leq k$. Then there exists a unitary cocycle $U$ for $E$ such that $X \cong X_{U}$.

Proof. (1) This follows immediately from [9, Theorem 2.1].

(2) Let $W: X_{e_{2}} \otimes_{A} X_{e_{1}} \rightarrow X_{e_{1}} \otimes_{A} X_{e_{2}}$ be the isomorphism determined by the composition of the multiplication map $X_{e_{2}} \otimes_{A} X_{e_{1}} \rightarrow X_{(1,1)}$ with the inverse of the multiplication map $X_{e_{1}} \otimes_{A} X_{e_{2}} \rightarrow X_{(1,1)}$. Write $E_{12}$ for the graph $\left(E^{0}, E_{1}^{1} E_{2}^{1}, r, s\right)$ and $E_{21}$ for the graph $\left(E^{0}, E_{2}^{1} E_{1}^{1}, r, s\right)$. By identifying each $X_{e_{i}}$ with $X\left(E_{i}\right)$, we can regard $W$ as an isomorphism $X\left(E_{2}\right) \otimes_{A} X\left(E_{1}\right) \rightarrow X\left(E_{1}\right) \otimes_{A} X\left(E_{2}\right)$. A straightforward calculation shows that there are isomorphisms $M_{i, j}: X\left(E_{i}\right) \otimes_{A} X\left(E_{j}\right) \rightarrow X\left(E_{i j}\right)$ such that $M_{i, j}\left(\delta_{e} \otimes \delta_{f}\right)=\delta_{e f}$ whenever $s(e)=r(f)$. So we obtain an isomorphism $\widetilde{W}: X\left(E_{21}\right) \rightarrow X\left(E_{12}\right)$ given by $\widetilde{W}=M_{1,2} \circ W \circ M_{2,1}^{-1}$. Since this is an isomorphism of Hilbert modules, it restricts to isomorphisms $\widetilde{W}(v, w): \delta_{v} \cdot X\left(E_{21}\right) \cdot \delta_{w} \rightarrow \delta_{v} \cdot X\left(E_{12}\right) \cdot \delta_{w}$. These spaces are canonically isomorphic to $\mathbb{C}^{v E_{2}^{1} E_{1}^{1} w}$ and $\mathbb{C}^{v E_{1}^{1} E_{2}^{1} w}$, and so $\widetilde{W}(v, w)$ determines a unitary $U_{1,2}(v, w) \in \mathcal{U}\left(\mathbb{C}^{v E_{2}^{1} E_{2}^{1} w}, \mathbb{C}^{v E_{1}^{1} E_{2}^{1} w}\right)$. 
The isomorphism $U_{1,2}: X\left(E_{2}\right) \otimes_{A} X\left(E_{1}\right) \rightarrow X\left(E_{1}\right) \otimes_{A} X\left(E_{2}\right)$ obtained from the maps $\left\{U_{1,2}(v, w)\right\}_{v, w}$ as in Lemma 4.3 satisfies $M_{1,2}\left(U_{1,2}\left(\delta_{f} \otimes \delta_{e}\right)\right)=\widetilde{W}\left(M_{2,1}\left(\delta_{f} \otimes \delta_{e}\right)\right)$ whenever $s(f)=r(e)$, and therefore $U_{1,2}=W$. So the uniqueness result [9, Theorem 2.2] shows that the product system $X$ is isomorphic to $X_{U}$ as claimed.

Our next result shows that for any row-finite $k$-graph $\Lambda$ with no sources, and for any 2 -cocycle on $\Lambda$, we obtain a unitary cocycle such that the twisted $k$-graph $C^{*}$-algebra coincides with the Cuntz-Nica-Pimsner algebra of the product system over $\mathbb{N}^{k}$ determined by the unitary cocycle.

Proposition 4.7. Let $\Lambda$ be a row-finite $k$-graph with no sources and suppose that $\phi$ : $\bigsqcup_{i<j \leq k} \Lambda^{e_{i}+e_{j}} \rightarrow \mathbb{T}$ is a cubical 2-cocycle. For $i \leq k$, let $E_{i}$ be the directed graph $E_{i}=$ $\left(\Lambda^{0}, \Lambda^{e_{i}}, r, s\right)$. Then $E=\left(E_{1}, \ldots, E_{k}\right)$ is a k-skeleton. There is a unitary cocycle $U=U^{\Lambda, \phi}$ for $E$ such that for all $v, w \in \Lambda^{0}$ and $1 \leq i<j \leq k$, and for all $e, e^{\prime} \in E_{i}^{1}$ and $f, f^{\prime} \in E_{j}^{1}$ satisfying ef $=f^{\prime} e^{\prime}$ in $\Lambda$, we have

$$
U_{i, j}(v, w)\left(\delta_{f^{\prime} e^{\prime}}\right)=\phi(e f) \delta_{e f} .
$$

Let $X_{U}$ be the product system associated to $U$ in Lemma [4.6. Then there is an isomorphism $\pi: C_{\phi}^{*}(\Lambda) \cong \mathcal{N} \mathcal{O}_{X_{U}}$ such that $\pi\left(p_{v}\right)=i_{0}\left(\delta_{v}\right)$ for all $v \in \Lambda^{0}$, and such that $\pi\left(s_{\lambda}\right) i_{e_{i}}\left(\delta_{\lambda}\right)$ for all $i \leq k$ and $\lambda \in \Lambda^{e_{i}}$.

Proof. The factorisation rules in $\Lambda$ determine bijections $f_{i j}: v \Lambda^{e_{i}} \Lambda^{e_{j}} w \rightarrow v \Lambda^{e_{i}+e_{j}} w$, and hence bijections $F_{i j}:=f_{i j}^{-1} \circ f_{j i}: v \Lambda^{e_{j}} \Lambda^{e_{i}} w \rightarrow v \Lambda^{e_{i}} \Lambda^{e_{j}} w$ for $i<j$, such that if $e, e^{\prime} \in$ $\Lambda^{e_{j}}=E_{i}^{1}$ and $f, f^{\prime} \in \Lambda^{e_{i}}=E_{j}^{1}$ satisfy $e f=f^{\prime} e^{\prime}$, then $F_{i j}(e f)=f^{\prime} e^{\prime}$. In particular, $E$ is a $k$-skeleton. The maps $F_{i j}$ induce a unitary (in fact a permutation matrix) $V_{i j}(v, w)$ : $\mathbb{C}^{v E_{j}^{1} E_{i}^{1} w} \rightarrow \mathbb{C}^{v E_{i}^{1} E_{j}^{1} w}$ for each $v, w \in E^{0}$, and post-composing $V_{i, j}(v, w)$ with the diagonal unitary $\operatorname{diag}(\phi(\lambda))_{\lambda \in v E_{i}^{1} E_{j}^{1} w}$ yields a unitary $U(v, w)_{i, j}$ satisfying the desired formula. If efg $\in E_{i}^{1} E_{j}^{1} E_{l}^{1}$ with $1 \leq l<j<i \leq k$, and if the factorisation rules give efg=f $f^{1} e^{1} g=$ $f^{1} g^{1} e^{2}=g^{2} f^{2} e^{2}$ and also $e f g=e g_{1} f_{1}=g_{2} e_{1} f_{1}=g_{2} f_{2} e_{2}$, then associativity of composition in $\Lambda$ gives $e^{2}=e_{2}, f^{2}=f_{2}$ and $g^{2}=g_{2}$ :

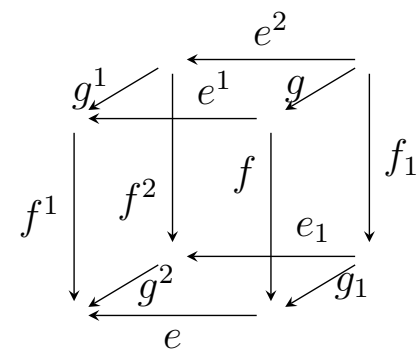

Quick calculations then give

$$
\left(U_{i, j} \otimes 1_{X_{l}}\right)\left(1_{X_{j}} \otimes U_{i, l}\right)\left(U_{j, l} \otimes 1_{X_{i}}\right)\left(\delta_{e} \otimes \delta_{f} \otimes \delta_{g}\right)=\phi(e f) \phi\left(e^{1} g\right) \phi\left(f^{1} g^{1}\right) \delta_{g^{2}} \otimes \delta_{f^{2}} \otimes \delta_{e^{2}},
$$

and

$$
\begin{aligned}
\left(1_{X_{i}} \otimes U_{j, l}\right)\left(U_{i, l} \otimes 1_{X_{j}}\right)\left(1_{X_{l}} \otimes U_{i, j}\right)\left(\delta_{e} \otimes \delta_{f} \otimes \delta_{g}\right) & =\phi(f g) \phi\left(e g_{1}\right) \phi\left(e_{1} f_{1}\right) \delta_{g_{2}} \otimes \delta_{f_{2}} \otimes \delta_{e_{2}} \\
& =\phi(f g) \phi\left(e g_{1}\right) \phi\left(e_{1} f_{1}\right) \delta_{g^{2}} \otimes \delta_{f^{2}} \otimes \delta_{e^{2}} .
\end{aligned}
$$

The cubical cocycle condition shows that $c(e f) c\left(e^{1} g\right) c\left(f^{1} g^{1}\right)=c(f g) c\left(e g_{1}\right) c\left(e_{1} f_{1}\right)$. Thus condition (4.2) holds when both sides are applied to an elementary tensor $\delta_{e} \otimes \delta_{f} \otimes \delta_{g}$ 
corresponding to a path efg $\in E_{i}^{1} E_{j}^{1} E_{l}^{1}$. Since these are the only elementary tensors of basis vectors that are nonzero, they span $X_{i} \otimes_{A} X_{j} \otimes_{A} X_{k}$, and therefore $U$ is a unitary cocycle for $E$ as claimed.

For $v \in \Lambda^{0}$, define $P_{v}:=i_{0}\left(\delta_{v}\right)$ and for $\lambda \in \Lambda^{e_{i}}$ define $S_{\lambda}:=i_{e_{i}}\left(\delta_{\lambda}\right)$. We claim that these form a Cuntz-Krieger $\phi$-representation of $\Lambda$ as in [18, Definition 7.4]. The $P_{v}$ are mutually orthogonal projections because the $\delta_{v}$ are. For $\lambda \in \Lambda^{e_{i}}$, we have

$$
S_{\lambda}^{*} S_{\lambda}=i_{e_{i}}\left(\delta_{\lambda}\right)^{*} i_{e_{i}}\left(\delta_{\lambda}\right)=i_{0}\left(\left\langle\delta_{\lambda}, \delta_{\lambda}\right\rangle_{A}\right)=i_{0}\left(\delta_{s}(\lambda)\right)=P_{s(\lambda)}
$$

which is condition (1) of [18, Definition 7.4].

To check condition (2), fix $1 \leq i<j \leq k$ and fix $\mu, \mu^{\prime} \in \Lambda^{e_{i}}$ and $\nu, \nu^{\prime} \in \Lambda^{e_{j}}$ such that $\mu \nu=\nu^{\prime} \mu^{\prime}$. Using (4.3) at the second and fifth equalities, and using the definition of $U_{r\left(\nu^{\prime}\right), s\left(\mu^{\prime}\right)}$ at the fourth, we calculate:

$$
\begin{aligned}
s_{\nu}^{\prime} s_{\mu}^{\prime} & =i_{e_{2}}\left(\delta_{\nu^{\prime}}\right) i_{e_{1}}\left(\delta_{\mu^{\prime}}\right)=i_{(1,1)}\left(U\left(\delta_{\nu^{\prime}} \otimes \delta_{\mu^{\prime}}\right)\right)=i_{(1,1)}\left(U_{r\left(\nu^{\prime}\right), s\left(\mu^{\prime}\right)}\left(\delta_{\nu^{\prime}} \otimes \delta_{\mu^{\prime}}\right)\right) \\
& =i_{(1,1)}\left(\phi(\mu \nu) \delta_{\mu} \otimes \delta_{\nu}\right)=\phi(\mu \nu) i_{e_{1}}\left(\delta_{\mu}\right) i_{e_{2}}\left(\delta_{\nu}\right)=\phi(\mu \nu) s_{\mu} s_{\nu} .
\end{aligned}
$$

Finally, to check condition (3), fix $v \in E^{0}$. Writing $\alpha: A \rightarrow \mathcal{K}\left(X_{i}\right)$ for the homomorphism implementing the left action, we have $\sum_{e \in v \Lambda} e_{i} \theta_{\delta_{e}, \delta_{e}}=\alpha\left(\delta_{v}\right)$, and so the Cuntz-Pimsner covariance condition gives $\sum_{e \in v \Lambda^{e_{i}}} S_{e} S_{e}^{*}=i^{\left(e_{i}\right)}\left(\sum_{e} \theta_{\delta_{e}, \delta_{e}}\right)=i_{0}\left(\delta_{v}\right)=P_{v}$. Thus the operators $\left\{P_{v}\right\}_{v \in \Lambda^{0}}$ and $\left\{S_{\lambda}\right\}_{\lambda \in \Lambda^{e_{i}}}$ form a Cuntz-Krieger $\phi$-representation of $\Lambda$ as claimed.

The universal property of $C_{\phi}^{*}(\Lambda)$ now gives a homomorphism $\pi: C_{\phi}^{*}(\Lambda) \rightarrow \mathcal{N} \mathcal{O}_{X}$ that carries $p_{v}$ to $P_{v}$ and $s_{\lambda}$ to $S_{\lambda}$ for $\lambda \in \bigsqcup_{i<k} \Lambda^{e_{i}}$. Since $A$ is spanned by the $\delta_{v}$ and each $X_{i}$ is spanned by $\left\{\delta_{\lambda}: \lambda \in \Lambda^{e_{i}}\right\}$ we see that the range of $\pi$ contains $C^{*}\left(i_{0}(A) \cup \bigcup_{i} i_{e_{i}}\left(X_{i}\right)\right)$. Since multiplication gives isomorphisms $X_{n} \cong X_{1}^{\otimes n_{1}} \otimes_{A} X_{2}^{\otimes n_{2}} \otimes_{A} \cdots \otimes_{A} X_{k}^{\otimes n_{k}}$, we see that

$$
i_{n}\left(X_{n}\right)=\overline{\operatorname{span}}\left\{S_{\lambda_{1}^{1}} \ldots S_{\lambda_{n_{1}}^{1}} S_{\lambda_{1}^{2}} \ldots S_{\lambda_{n_{2}}^{2}} \ldots S_{\lambda_{1}^{k}} \ldots S_{\lambda_{n_{k}}^{k}}: \lambda_{j}^{i} \in \Lambda^{e_{i}}\right\}
$$

and so is contained in the image of $\pi$. Since $i_{0}$ is injective [32, Lemma 3.5 and Theorem 4.1], the $P_{v}$ are all nonzero. Using [19, Theorem 4.15 and Proposition 5.7] we see that there is an isomorphism $\omega: C_{\phi}^{*}(\Lambda) \cong C^{*}(\Lambda, c)$, for some categorical 2-cocycle $c$, such that $\omega$ carries $p_{v}$ to $p_{v}$ for each $v \in \Lambda^{0}$, and then an application of the gauge-invariant uniqueness theorem [19, Corollary 7.7] shows that $\pi \circ \omega^{-1}$ is injective, and therefore $\pi$ is injective. So $C_{\phi}^{*}(\Lambda) \cong \mathcal{N O}_{Y^{0}}$ as claimed.

Lemma 4.6 also allows us to construct homotopies of product systems using continuous paths of unitary cocycles.

Definition 4.8. Let $E=\left(E_{1}, \ldots, E_{k}\right)$ be a $k$-skeleton. A continuous path of unitary cocycles for $E$ is a family

$$
\left\{U_{i, j}^{t}(v, w): v, w \in E^{0}, 1 \leq i<j \leq k, t \in[0,1]\right\}
$$

such that for each $t$, the family $\left\{U_{i, j}^{t}(v, w): v, w \in E^{0}, 1 \leq i<j \leq k\right\}$ is a unitary cocycle for $E$, and such that for each $i<j$ and each $v, w \in E^{0}$, the function $t \mapsto U_{i, j}^{t}(v, w)$ is a continuous function from $[0,1]$ to $\mathcal{U}\left(\mathbb{C}^{v E_{j}^{1} E_{i}^{1} w}, \mathbb{C}^{v E_{i}^{1} E_{j}^{1} w}\right)$.

Proposition 4.9. Let $E=\left(E_{1}, \ldots, E_{k}\right)$ be a $k$-skeleton, and suppose that the family $U=\left\{U_{i, j}^{t}(v, w): v, w \in E^{0}, 1 \leq i<j \leq k, t \in[0,1]\right\}$ is a continuous path of unitary cocycles for $E$. Let $A=C_{0}\left(E^{0}\right)$, and for each $i$, let $X_{i}=X\left(E_{i}\right)$ the graph bimodule for $E_{i}$. 
Define $B:=C([0,1], A)$ and for each $i$, define $Y_{i}:=C\left([0,1], X_{i}\right)$ regarded as a Hilbert $B$ bimodule under the actions $(f \cdot F \cdot g)(t)=f(t) \cdot F(t) \cdot g(t)$ and $\langle F, G\rangle_{B}(t)=\langle F(t), G(t)\rangle_{A}$. Then there is a unique product system $Y$ over $\mathbb{N}^{k}$ with coefficient algebra $B$ such that: $Y_{e_{i}}=Y_{i}$ for $i \leq k$; for all $1 \leq i<j \leq k$, we have $Y_{e_{i}+e_{j}}=Y_{i} \otimes_{B} Y_{j}$; and multiplication in $Y$ satisfies

$$
(F G)(t)=F(t) \otimes_{A} G(t) \quad \text { and } \quad(G F)(t)=U_{i, j}^{t}(G(t) \otimes F(t))
$$

whenever $1 \leq i<j \leq k, F \in Y_{i}, G \in Y_{j}$ and $t \in[0,1]$. The product system $Y$ is a homotopy of product systems from $X_{U^{0}}$ to $X_{U^{1}}$.

Proof. Since each $U_{i, j}^{t}$ gives an isomorphism $X_{j} \otimes_{A} X_{i} \rightarrow X_{i} \otimes_{A} X_{j}$, and $U$ is a continuous path of unitary cocycles, the map $G \otimes F \mapsto\left(t \mapsto U_{i, j}^{t}(G(t) \otimes F(t))\right)$ determines an isomorphism $U_{i, j}: Y_{j} \otimes_{B} Y_{i} \rightarrow Y_{i} \otimes_{B} Y_{j}$. Since the $U_{i, j}^{t}$ satisfy the cocycle identity, so do the induced isomorphisms $U_{i, j}$. So the first statement follows from [9, Theorem 2.1].

The formulas for the actions show immediately that $Y$ is nondegenerate, and is fibred over $[0,1]$. Thus, it suffices to show that $Y^{t}=X_{U^{t}}$. Let $I_{t}$ be the ideal of $B$ generated by $C_{0}([0,1] \backslash\{t\}) \subseteq C([0,1])$. For $F, G \in Y_{i}$, we have $F+Y_{i} \cdot I_{t}=G+Y_{i} \cdot I_{t}$ if and only if $F(t)=G(t)$. Routine calculations then show that the map $\eta_{t}: F+Y_{i} \cdot I_{t} \mapsto F(t)$ is a bimodule morphism (in fact an isomorphism) from $Y_{i}^{t}$ to $X_{i}$. Since each $Y_{i}$ is fibred over $[0,1]$ we see that $\left(Y_{i} \otimes_{B} Y_{j}\right) \cdot I_{t}=\left(Y_{i} \cdot I_{t}\right) \otimes_{B}\left(Y_{j} \cdot I_{t}\right)$ for all $i, j$ and $t$, and that $\eta_{t}$ induces an isomorphism (also denoted $\eta_{t}$ ) from $Y_{i}^{t} \otimes_{A} Y_{j}^{t}$ to $X_{i} \otimes_{A} X_{j}$. These isomorphisms satisfy

$$
\begin{aligned}
U_{i, j}^{t}\left(\eta_{t}\left(\left(G+Y_{j} \cdot I_{t}\right) \otimes\left(F+Y_{i} \cdot I_{t}\right)\right)\right) & =U_{i, j}^{t}(G(t) \otimes F(t))=\left(U_{i, j}(G \otimes F)\right)(t) \\
& =\eta_{t}\left(U_{i, j}(G \otimes F)+\left(Y_{i} \otimes_{B} Y_{j}\right) \cdot I_{t}\right),
\end{aligned}
$$

and so it follows from the uniqueness theorem [9, Theorem 2.2] that the product system $Y^{t}$ is isomorphic to $X_{U^{t}}$. In particular $Y^{0} \cong X_{U^{0}}$ and $Y^{1} \cong X_{U^{1}}$.

Corollary 4.10. Let $E=\left(E_{1}, \ldots, E_{k}\right)$ be a k-skeleton. If $U=\left\{U_{i, j}^{t}(v, w)\right\}$ is a continuous path of unitary cocycles for $E$, then $K_{*}\left(\mathcal{N O}_{X_{U^{0}}}\right) \cong K_{*}\left(\mathcal{N O}_{X_{U^{1}}}\right)$. In particular, suppose that $\Lambda_{1}$ and $\Lambda_{2}$ are $k$-graphs with the same skeleton and that $\phi_{l}: \bigsqcup_{i<j \leq k} \Lambda_{l}^{e_{i}+e_{j}} \rightarrow \mathbb{T}$ is a cubical cocycle for $i=1,2$. If there is a continuous path of unitary cocycles from $U_{\phi_{1}}$ to $U_{\phi_{2}}$, then $K_{*}\left(C_{\phi_{1}}^{*}\left(\Lambda_{1}\right)\right) \cong K_{*}\left(C_{\phi_{2}}^{*}\left(\Lambda_{2}\right)\right)$.

Proof. Proposition 4.9 gives a homotopy of product systems from $X_{U^{0}}$ to $X_{U^{1}}$, and then Theorem 3.7 implies that $K_{*}\left(\mathcal{N O}_{X_{U^{0}}}\right) \cong K_{*}\left(\mathcal{N O}_{X_{U^{1}}}\right)$ as required. The final statement follows from the first statement and Proposition 4.7 .

The next result demonstrates that Corollary 4.10 is particularly useful when $k=2$.

Proposition 4.11. Let $E=\left(E_{1}, E_{2}\right)$ be a 2-skeleton. Then the space of unitary cocycles for $E$ is path-connected in the sense that for any two unitary cocycles $V, W$ for $E$ there is a continuous path $U^{t}$ of unitary cocycles for $E$ such that $U^{0}=V$ and $U^{1}=W$.

Proof. Fix $v, w \in E^{0}$. The space

$$
\mathcal{U}\left(\mathbb{C}^{v E_{j}^{1} E_{i}^{1} w}, \mathbb{C}^{v E_{i}^{1} E_{j}^{1} w}\right)
$$

is homeomorphic to the finite-dimensional unitary group $\mathcal{U}_{\left|v \Lambda^{(1,1)} w\right|}$, and therefore pathconnected. So we can choose a continuous path $U_{1,2}^{t}(v, w)$ in $\mathcal{U}\left(\mathbb{C}^{v E_{j}^{1} E_{i}^{1} w}, \mathbb{C}^{v E_{i}^{1} E_{j}^{1} w}\right)$ such that $U_{1,2}^{0}(v, w)=V_{1,2}(v, w)$ and such that $U_{1,2}^{1}(v, w)=W_{1,2}(v, w)$. Since $k=2$, the 
resulting isomorphisms $U_{i, j}^{t}: X\left(E_{j}\right) \otimes_{A} X\left(E_{i}\right) \rightarrow X\left(E_{i}\right) \otimes_{A} X\left(E_{j}\right)$ indexed by $1 \leq i<j \leq$ 2 all vacuously satisfy the condition (4.2) (see Remark 4.5). So the $U_{i, j}^{t}(v, w)$ constitute a continuous path of unitary cocycles for $E$.

Corollary 4.12. Let $E=\left(E_{1}, E_{2}\right)$ be a 2-skeleton. Suppose that $X^{0}$ and $X^{1}$ are product systems such that $X_{j}^{n} \cong X\left(E_{j}\right)$ for all $n \in\{0,1\}, j \in\{1,2\}$. Then $K_{*}\left(\mathcal{N O}_{X^{0}}\right) \cong$ $K_{*}\left(\mathcal{N O}_{X^{1}}\right)$.

Proof. By Lemma 4.6, there are unitary cocycles $U^{n}$ for $E$ such that $X^{n} \cong X_{U^{n}}$ for $n=0,1$. Proposition 4.11 shows that there is a continuous path of unitaries connecting $U^{0}$ and $U^{1}$. Hence Corollary 4.10 gives the result.

We can now prove the main theorem of the section.

Proof of Theorem 4.1. For $i=1,2$ let $E_{i}$ be the graph $\left(\Lambda^{0}, \Lambda^{e_{i}}, r, s\right)$. Proposition 4.7 shows that there are product systems $X^{\Lambda, \phi}$ and $X^{\Gamma, \psi}$ over $\mathbb{N}^{2}$ with coefficient algebra $C_{0}\left(E^{0}\right)$ such that $X_{e_{i}}^{\Lambda, \phi} \cong X_{e_{i}}^{\Gamma, \psi} \cong X\left(E_{i}\right)$ for $i=1,2$ and such that $\mathcal{N} \mathcal{O}_{X^{\Lambda, \phi}} \cong C_{\phi}^{*}(\Lambda)$ and $\mathcal{N} \mathcal{O}_{X^{\Gamma, \psi}} \cong C_{\psi}^{*}(\Gamma)$. So the result follows from Corollary 4.12.

Remark 4.13. Let $E=\left(E_{1}, E_{2}\right)$ be a 2-skeleton. By [15, Section 6] (see also [9, Remark 2.3]), any range-and-source-preserving bijection $E_{2}^{1} E_{1}^{1} \rightarrow E_{1}^{1} E_{2}^{1}$ determines a 2graph with skeleton $E$. In particular, there is at least one such 2-graph $\Lambda$. Let $M_{1}$ and $M_{2}$ be the $E^{0} \times E^{0}$ adjacency matrices of $E_{1}$ and $E_{2}$; that is $M_{i}(v, w)=\left|v E_{i}^{1} w\right|$. We regard the transpose matrices $M_{i}^{T}$ as homomorphisms $M_{i}^{T}: \mathbb{Z} E^{0} \rightarrow \mathbb{Z} E^{0}$, and then we obtain homomorphisms

$$
\left(1-M_{1}^{t}, 1-M_{2}^{t}\right): \mathbb{Z} E^{0} \oplus \mathbb{Z} E^{0} \rightarrow \mathbb{Z} E^{0} \quad \text { and } \quad\left(\begin{array}{c}
M_{2}^{t}-1 \\
1-M_{1}^{t}
\end{array}\right): \mathbb{Z} E^{0} \rightarrow \mathbb{Z} E^{0} \oplus \mathbb{Z} E^{0}
$$

Evans' calculation [4, Proposition 3.16] of the $K$-theory of the $C^{*}$-algebra of a row-finite 2-graph shows that

$$
\begin{aligned}
& K_{0}\left(C^{*}(\Lambda)\right) \cong \operatorname{coker}\left(1-M_{1}^{t}, 1-M_{2}^{t}\right) \oplus \operatorname{ker}\left(\begin{array}{c}
M_{2}^{t}-1 \\
1-M_{1}^{t}
\end{array}\right), \quad \text { and } \\
& K_{1}\left(C^{*}(\Lambda)\right) \cong \operatorname{ker}\left(1-M_{1}^{t}, 1-M_{2}^{t}\right) / \operatorname{image}\left(\begin{array}{c}
M_{2}^{t}-1 \\
1-M_{1}^{t}
\end{array}\right) .
\end{aligned}
$$

Combining this with Proposition 4.7 and Corollary 4.12, we deduce that if $X$ is any product system over $\mathbb{N}^{2}$ with coefficient algebra $C_{0}\left(E^{0}\right)$ such that $X_{e_{i}} \cong X\left(E_{i}\right)$ for $i=1,2$,

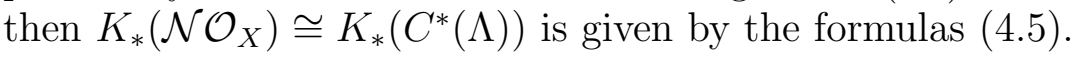

We can also say a little about the situation of single-vertex $k$-graphs. Although we do not have an entirely satisfactory result in this situation, we indicate what we can say since there has been some interest (see Corollary 5.8, Remark 5.9, and the following paragraph of [1]) in deciding whether the $K$-theory of the $C^{*}$-algebra of a 1 -vertex $k$-graph is independent of the factorisation rules.

Fix $k \geq 0$ and integers $n_{1}, \ldots, n_{k} \geq 1$. Suppose that, for each $1 \leq i<j \leq k$, we have a unitary transformation $U_{i, j}: \mathbb{C}^{n_{j}} \otimes \mathbb{C}^{n_{i}} \rightarrow \mathbb{C}^{n_{i}} \otimes \mathbb{C}^{n_{j}}$. We say that the system $U_{i, j}$ is a unitary cocycle for $\left(n_{1}, \ldots, n_{k}\right)$ if, for all $1 \leq i<j<l \leq k$, we have

$$
\left(U_{i, j} \otimes 1_{n_{l}}\right)\left(1_{n_{j}} \otimes U_{i, l}\right)\left(U_{j, l} \otimes 1_{n_{i}}\right)=\left(1_{n_{i}} \otimes U_{j, l}\right)\left(U_{i, l} \otimes 1_{n_{j}}\right)\left(1_{n_{l}} \otimes U_{i, j}\right) .
$$


A continuous path of unitary cocycles for $\left(n_{1}, \ldots, n_{k}\right)$ is a family $\left\{U_{i, j}^{t}: t \in[0,1], 1 \leq i<\right.$ $j \leq k\}$ of unitary cocycles for $\left(n_{1}, \ldots, n_{k}\right)$ such that the map $t \mapsto U_{i, j}^{t}$ is continuous for each $1 \leq i<j \leq k$.

Example 4.14. Let $\Lambda$ be a $k$-graph with a single vertex $v$ and satisfying $\left|\Lambda^{e_{i}}\right|=n_{i}$ for $1 \leq i \leq k$. Let $\phi: \bigsqcup_{i \neq j} \Lambda^{e_{i}+e_{j}} \rightarrow \mathbb{T}$ be a cubical 2-cocycle. Identifying $\mathbb{C}^{n_{i}} \otimes \mathbb{C}^{n_{j}}$ with $\mathbb{C}^{\Lambda^{e_{i}} \Lambda^{e_{j}}}$ for all $i \neq j$, we obtain from Proposition 4.7 a unitary cocycle $U^{\Lambda, \phi}$ for $\left(n_{1}, \ldots, n_{k}\right)$.

Corollary 4.15. Fix integers $k \geq 0$ and $n_{1}, \ldots, n_{k} \geq 1$.

(1) Let $\Lambda_{1}$ and $\Lambda_{2}$ be single-vertex $k$-graphs such that $\left|\Lambda_{1}^{e_{i}}\right|=\left|\Lambda_{2}^{e_{i}}\right|=: n_{i}$ for all $i$. If there is a continuous path of unitary cocycles for $\left(n_{1}, \ldots, n_{k}\right)$ from $U^{\Lambda_{1}, 1}$ to $U^{\Lambda_{2}, 1}$, then $K_{*}\left(C^{*}\left(\Lambda_{1}\right)\right) \cong K_{*}\left(C^{*}\left(\Lambda_{2}\right)\right)$.

(2) If the space of all unitary cocycles for $\left(n_{1}, \ldots, n_{k}\right)$ is path-connected, then we have $K_{*}\left(C^{*}\left(\Lambda_{1}, c_{1}\right)\right) \cong K_{*}\left(C^{*}\left(\Lambda_{2}, c_{2}\right)\right)$ for any single vertex $k$-graphs $\Lambda_{i}$ with $\left|\Lambda_{i}^{e_{j}}\right|=n_{j}$ and any categorical 2-cocycles $c_{i}$ on $\Lambda_{i}$.

Proof. This follows immediately from Corollary 4.10.

Unfortunately, we do not know whether the space of unitary cocycles for $\left(n_{1}, \ldots, n_{k}\right)$ is connected: certainly given any two unitary cocycles $U$ and $V$, we can find continuous paths $W_{i, j}^{t}$ from $U_{i, j}$ to $V_{i, j}$ for all $i, j$, but it is not at all clear that these can be chosen so that, for each $t,\left\{W_{i, j}^{t}: 1 \leq i<j \leq k\right\}$ satisfies the cocycle condition (4.6). This seems closely related to the question of whether the space of solutions to the Yang-Baxter equation is path-connected (see [34]).

Remark 4.16. More generally, if $E=\left(E_{1}, \ldots, E_{k}\right)$ is a $k$-skeleton such that the space of unitary cocycles for $E$ is path-connected, then for any two $k$-graphs $\Lambda$ and $\Gamma$ with common skeleton $\Lambda^{e_{i}}=E_{i}=\Gamma^{e_{i}}$, and any cubical cocycles $\phi$ for $\Lambda$ and $\psi$ for $\Gamma$, we have $K_{*}\left(C_{\phi}^{*}(\Lambda)\right) \cong K_{*}\left(C_{\psi}^{*}(\Gamma)\right)$. Again, since each $\mathbb{C}^{v E_{j} E_{i} w}$ is finite dimensional, for each $u, v, i, j$ we can find a continuous path of unitaries from $U_{i, j}^{\Lambda, \phi}(u, v)$ to $U_{i, j}^{\Gamma, \phi}(u, v)$, but it is not at all clear that these can be chosen to satisfy the cocycle identity (4.2).

\section{REFERENCES}

[1] S. Barlak, T. Omland and N. Stammeier, On the $K$-theory of $C^{*}$-algebras arising from integral dynamics, Ergodic Theory Dynam. Systems 38 (2018), 832-862.

[2] L.O. Clark, A. an Huef and A. Sims, AF-embeddability of 2-graph algebras and quasidiagonality of $k$-graph algebras, J. Funct. Anal. 271 (2016), 958-991.

[3] G.A. Elliott, On the $K$-theory of the $C^{*}$-algebra generated by a projective representation of a torsionfree discrete abelian group, Monogr. Stud. Math., 17, Operator algebras and group representations, Vol. I (Neptun, 1980), 157-184, Pitman, Boston, MA, 1984.

[4] D.G. Evans, On the K-theory of higher-rank graph $C^{*}$-algebras, New York J. Math. 14 (2008), 1-31.

[5] J. Fletcher, Iterating the Cuntz-Nica-Pimsner construction for product systems, PhD thesis, University of Wollongong, 2017.

[6] J. Fletcher, Iterating the Cuntz-Nica-Pimsner construction for compactly aligned product systems, New York J. Math. 24 (2018), 739-814.

[7] N.J. Fowler, Discrete product systems of Hilbert bimodules, Pacific J. Math. 204 (2002), 335-375.

[8] N.J. Fowler and I. Raeburn, The Toeplitz algebra of a Hilbert bimodule, Indiana Univ. Math. J. 48 (1999), 155-181.

[9] N.J. Fowler and A. Sims, Product systems over right-angled Artin semigroups, Trans. Amer. Math. Soc. 354 (2002), 1487-1509. 
[10] E. Gillaspy, K-theory and homotopies of 2-cocycles on higher-rank graphs, Pacific J. Math. 278 (2015), 407-426.

[11] E. Gillaspy and J. Wu, Isomorphism of the cubical and categorical cohomology groups of a higher-rank graph , preprint 2018 (arXiv:1807.02245 [math.OA]).

[12] R. Hazlewood, I. Raeburn, A. Sims, and S.B.G. Webster, Remarks on some fundamental results about higher-rank graphs and their $C^{*}$-algebras, Proc. Edinburgh Math. Soc. 56 (2013), 575-597.

[13] M. Jimbo, Introduction to the Yang-Baxter equation, Int. J. Modern Phys. A. 4 (1989), 3759-3777.

[14] T. Katsura, On $C^{*}$-algebras associated with $C^{*}$-correspondences, J. Funct. Anal. 217 (2004), 366401.

[15] A. Kumjian and D. Pask, Higher rank graph $C^{*}$-algebras, New York J. Math. 6 (2000), 1-20.

[16] A. Kumjian, D. Pask and I. Raeburn, Cuntz-Krieger algebras of directed graphs, Pacific J. Math. 184 (1998), 161-174.

[17] A. Kumjian, D. Pask and A. Sims, Generalised morphisms of k-graphs: k-morphs, Trans. Amer. Math. Soc. 363 (2011), 2599-2626.

[18] A. Kumjian, D. Pask and A. Sims, Homology for higher-rank graphs and twisted $C^{*}$-algebras, J. Funct. Anal. 263 (2012), 1539-1574.

[19] A. Kumjian, D. Pask and A. Sims, On twisted higher-rank graph $C^{*}$-algebras, Trans. Amer. Math. Soc. 367 (2015), 5177-5216.

[20] A. Kumjian, D. Pask and A. Sims, On the K-theory of twisted higher-rank-graph $C^{*}$-algebras, J. Math. Anal. Appl. 401 (2013), 104-113.

[21] A. Kumjian, D. Pask and A. Sims, Graded $C^{*}$-algebras, graded $K$-theory, and twisted P-graph $C^{*}$ algebras, J. Operator Theory 80 (2018), 295-348.

[22] E.C. Lance, Hilbert $C^{*}$-modules, A toolkit for operator algebraists, Cambridge University Press, Cambridge, 1995, x+130.

[23] D. Pask, A. Sierakowski and A. Sims, Real rank and topological dimension of higher-rank graph algebras, Indiana Univ. Math. J. 66 (2017), 2137-2168.

[24] M.V. Pimsner, A class of $C^{*}$-algebras generalizing both Cuntz-Krieger algebras and crossed products by Z, Fields Inst. Commun., 12, Free probability theory (Waterloo, ON, 1995), 189-212, Amer. Math. Soc., Providence, RI, 1997.

[25] I. Raeburn, Graph algebras, Published for the Conference Board of the Mathematical Sciences, Washington, DC, 2005, vi+113.

[26] I. Raeburn and A. Sims, Product systems of graphs and the $C^{*}$-algebras of higher-rank graphs, J. Operator Th. 53 (2005), 399-429.

[27] I. Raeburn and D.P. Williams, Morita equivalence and continuous-trace $C^{*}$-algebras, American Mathematical Society, Providence, RI, 1998, xiv +327.

[28] D.I. Robertson and A. Sims, Simplicity of $C^{*}$-algebras associated to higher-rank graphs, Bull. Lond. Math. Soc. 39 (2007), 337-344.

[29] G. Robertson and T. Steger, Affine buildings, tiling systems and higher rank Cuntz-Krieger algebras, J. reine angew. Math. 513 (1999), 115-144.

[30] A. Sims, Gauge-invariant ideals in the $C^{*}$-algebras of finitely aligned higher-rank graphs, Canad. J. Math. 58 (2006), 1268-1290.

[31] A. Sims, Relative Cuntz-Krieger algebras of finitely aligned higher-rank graphs, Indiana Univ. Math. J. 55 (2006), 849-868.

[32] A. Sims and T. Yeend, $C^{*}$-algebras associated to product systems of Hilbert bimodules, J. Operator Theory 64 (2010), 349-376.

[33] D.P. Williams, Crossed products of $C^{*}$-algebras, American Mathematical Society, Providence, RI, 2007, xvi+528.

[34] D. Yang, The interplay between k-graphs and the Yang-Baxter equation, J. Algebra 451 (2016), 494-525.

(J. Fletcher) School of Mathematics and Statistics, Victoria University of Wellington, PO Box 600, Wellington 6140, New Zealand

E-mail address: jef336@uowmail.edu.au 
(E. Gillaspy) Department of Mathematical Sciences, University of Montana, 32 Campus Drive \#0864, Missoula, MT 59812, USA

E-mail address: elizabeth.gillaspy@mso.umt.edu

(A. Sims) School of Mathematics and Applied Statistics, University of Wollongong, Northfields Ave Wollongong NSW 2522, Australia

E-mail address: asims@uow.edu.au 